\title{
¿Optimal Initial Excitations of Decadal Modification of the Atlantic Meridional Overturning Circulation under the Prescribed Heat and Freshwater Flux Boundary Conditions
}

\author{
ZIQING ZU \\ Key Laboratory of Research on Marine Hazards Forecasting, National Marine Environmental Forecasting \\ Center, Beijing, China \\ Mu Mu \\ Key Laboratory of Ocean Circulation and Wave, Institute of Oceanology, Chinese Academy of Sciences, and \\ Laboratory for Ocean and Climate Dynamics, Qingdao National Laboratory for Marine Science and \\ Technology, Qingdao, China \\ HENK A. DiJKstra \\ Institute for Marine and Atmospheric Research Utrecht, Department of Physics and Astronomy, Utrecht \\ University, Utrecht, Netherlands
}

(Manuscript received 2 June 2015, in final form 4 March 2016)

\begin{abstract}
Within a three-dimensional ocean circulation model, the nonlinear optimal initial perturbations (NOIP) of sea surface salinity (SSS) and sea surface temperature (SST) to excite variability in the Atlantic meridional overturning circulation (AMOC) were obtained under prescribed heat and freshwater flux boundary conditions, using the conditional nonlinear optimal perturbation (CNOP) method. After 10 years, the optimal SSS and SST perturbations lead to reductions of the AMOC by 3.6 and $2.5 \mathrm{~Sv}\left(1 \mathrm{~Sv}=10^{6} \mathrm{~m}^{3} \mathrm{~s}^{-1}\right)$, respectively, followed by multidecadal oscillations with a period of about 50 years. During the first 30 years, nonlinear processes have an important influence on the AMOC strength: convection strengthens the AMOC during years 0-2, zonal density advection promotes the slowdown of the AMOC during years 7-20, and meridional density advection inhibits the slowdown of meridional velocities in the upper ocean during years 5-18. The linear optimal initial perturbation (LOIP) was also computed using the first singular vector (FSV) method. For SSS perturbations with an amplitude of $0.5 \mathrm{psu}$, the LOIP will cause an underestimation of the amplitude of the multidecadal AMOC variability by about $1 \mathrm{~Sv}$, compared to that induced by the NOIP. This underestimation will become more significant as the amplitudes of SSS perturbations increase.
\end{abstract}

\section{Introduction}

The Atlantic meridional overturning circulation (AMOC) presents a broad spectrum of variability from seasonal to millennium time scales (Hobbs and Willis 2012). The Atlantic multidecadal variability (AMV) involves (Kushnir 1994) the variations of sea surface

○ Denotes Open Access content.

Corresponding author address: $\mathrm{Mu} \mathrm{Mu}$, Key Laboratory of Ocean Circulation and Wave, Institute of Oceanology, Chinese Academy of Sciences, 7 Nanhai Road, Qingdao 266071, China. E-mail: mumu@qdio.ac.cn. temperature (SST), and exerts an influence on the local and global climate (Enfield et al. 2001; Wang et al. 2009). A better understanding of the AMV is important to explain historical climate change and for the interpretation of projections of future climate change.

Coupled general circulation models have been used to simulate the multidecadal variability in the Atlantic. The relevant processes were then deduced from (a mainly statistical) analysis of the different fields. From these simulation studies, multidecadal variability has been attributed to 1 ) variability in the atmospheric forcing (Eden and Jung 2001), 2) coupled ocean-atmosphere interaction processes (Timmermann et al. 1998), 3) the coupling between the Atlantic and the Arctic (Jungclaus et al. 2005), 
and 4) the excitation of internal ocean variability (Delworth et al. 1995; Delworth and Mann 2000). All of the mechanisms share the fact that variability of the AMOC is involved.

Most of the conceptual models of the multidecadal variability (Chen and Ghil 1995; Colin de Verdière and Huck 1999; Huck and Vallis 2001; Dijkstra et al. 2006) focus on the fourth mechanism. In the limit of zero atmospheric damping, sustained oscillations are found in these conceptual models. However, this oscillatory behavior is damped under more realistic atmospheric damping conditions. In this case, it can be excited by stochastic forcing, for example, in the heat flux (Timmermann and Lohmann 2000; Monahan et al. 2008) or possibly by instantaneous thermohaline anomalies (Sun et al. 2005; Sévellec et al. 2009).

It is therefore important to understand which perturbation patterns will optimally excite the multidecadal variability in the AMOC. Several optimization methods have been used to yield the optimal initial anomalies that can lead to the largest modification of the AMOC (Farrell and Ioannou 1996; Mu et al. 2004; Hawkins and Sutton 2009). The first singular vector (FSV) method has been widely used (Farrell and Ioannou 1996) to produce the linear optimal initial perturbation (LOIP). In the tangent linear model, the LOIP grows fastest and causes the largest modification of the AMOC, which has been attributed to the nonnormal interaction of the eigenvectors (Alexander and Monahan 2009; Zanna et al. 2012). Using the FSV method and a box model, Tziperman and Ioannou (2002) found that multidecadal variability could be induced by the nonnormal interaction of two eigenvectors with different decay time scales. Using the ocean general circulation model Océan Parallélisé (OPA) 8.2 in a realistic configuration, Sévellec and Fedorov (2015) also calculated the LOIP by defining a linear objective function, rather than a quadratic one required by FSV. They found that the LOIP excites multidecadal oscillations under prescribed heat and freshwater flux boundary conditions.

The investigation of the optimal initial perturbation can also produce upper bounds of the AMOC response. For the LOIP of sea surface salinity (SSS) with a typical amplitude of Great Salinity Anomaly (GSA) events (Belkin et al. 1998), the AMOC slowed down by $1.3 \mathrm{~Sv}$ $\left(1 \mathrm{~Sv}=10^{6} \mathrm{~m}^{3} \mathrm{~s}^{-1}\right)$ at most (Sévellec and Fedorov 2015). Zanna et al. (2012) found that the AMOC would decrease by $1.7 \mathrm{~Sv}$ at most, if superimposing the LOIP of density with an amplitude of $0.02 \mathrm{~kg} \mathrm{~m}^{-3}$.

When perturbations grow, however, nonlinear processes eventually affect their evolution (Dijkstra 2007) because of the changing background flow, and the LOIP is no longer the fastest-growing perturbation. Therefore, the condition nonlinear optimal perturbation (CNOP) methodology was used to determine the nonlinear optimal initial perturbation (NOIP) patterns that optimally grow in the fully nonlinear flow (Mu et al. 2004; Sun et al. 2005; Zu et al. 2013). Using the CNOP method and a box model, Mu et al. (2004) found that the difference between the NOIP and LOIP increases with increasing amplitude of the perturbations. A nonlinear advective feedback was proposed to explain the difference between the nonlinear responses to two types of CNOPs.

Within a three-dimensional ocean circulation model of the North Atlantic, $\mathrm{Zu}$ et al. (2013) obtained two kinds NOIPs, corresponding to the global and local maxima of the objective functions, respectively. They found that these two kinds of the perturbations led to significantly different multidecadal variations of the AMOC, which was explained through a nonlinear advective feedback between density and velocity anomalies. However, they used mixed boundary conditions (restoring for temperature and prescribed flux for salinity), and the anomalies did not induce oscillatory AMOC variations because of the strong damping of SST anomalies. According to Sévellec et al. (2009), the LOIP induced large differences in the AMOC response under different boundary conditions.

In this paper, the NOIPs are determined under the prescribed heat and freshwater flux boundary conditions and the physics of the excitation of the multidecadal variability is analyzed. The paper is organized as follows. Section 2 introduces the model and methods. Section 3 presents the NOIP and the multidecadal variability it excites, and in section 4, the linear and nonlinear mechanisms of the AMOC response are addressed. Section 5 addresses the differences between the NOIP and LOIP, and a summary and discussion are provided in section 6 .

\section{Methodology}

\section{a. The model}

The three-dimensional ocean model used here is the thermohaline circulation model (THCM), as presented in Dijkstra et al. (2001) and de Niet et al. (2007). The model uses hydrostatic and primitive equations in spherical coordinates. The equations are discretized using a control volume method spatially and a fully implicit scheme temporally, which allows for a relatively large time step, compared to explicit methods. The discretized algebraic equations are solved by the Newton-Raphson method, which also yields the tangent linear matrix and the associated adjoint matrix explicitly (these matrices are used to 
TABLE 1. Standard values of parameters used in THCM.

\begin{tabular}{ll}
\hline \hline Horizontal friction coefficient & $5.6 \times 10^{5} \mathrm{~m}^{2} \mathrm{~s}^{-1}$ \\
Vertical friction coefficient & $1.0 \times 10^{-3} \mathrm{~m}^{2} \mathrm{~s}^{-1}$ \\
Horizontal tracer diffusive coefficient & $1.2 \times 10^{3} \mathrm{~m}^{2} \mathrm{~s}^{-1}$ \\
Vertical tracer diffusive coefficient & $1.2 \times 10^{-4} \mathrm{~m}^{2} \mathrm{~s}^{-1}$ \\
Vertical convective coefficient & $3.0 \times 10^{-2} \mathrm{~m}^{2} \mathrm{~s}^{-1}$ \\
Thermal expansion coefficient & $1.0 \times 10^{-4}$ \\
Haline expansion coefficient & $7.6 \times 10^{-4}$ \\
Restoring time scales $\left(\tau_{T}, \tau_{S}\right)$ & 75 days \\
Amplitude of $\operatorname{SST}_{\text {fix }}\left(A_{T}\right)$ & $15^{\circ} \mathrm{C}$ \\
${\text { Amplitude of } \operatorname{SSS}_{\text {fix }}\left(A_{S}\right)}$ & $0.5 \mathrm{psu}$ \\
\hline
\end{tabular}

reduce the CNOP computations during optimization). THCM has been used extensively to study the sensitivity and multidecadal variability of the AMOC (Dijkstra et al. 2006; Huisman et al. 2010).

An idealized configuration in THCM was defined in which the depth of the basin studied was uniformly $4000 \mathrm{~m}$ and the domain of integration used was $\left[74^{\circ} \mathrm{W}\right.$, $\left.10^{\circ} \mathrm{W}\right] \times\left[10^{\circ} \mathrm{N}, 74^{\circ} \mathrm{N}\right]$. The resolution chosen was $4^{\circ} \times 4^{\circ}$ horizontally and $250 \mathrm{~m}$ vertically. The time step was variable: a short time step of 7 days for the fast-adjustment stage and a long time step of 21 days for the slow-evolution stage. The wind forcing was neglected. Several important parameters used in THCM were listed in Table 1 and other parameters were set as in de Niet et al. (2007).

To reach a steady state, restoring boundary conditions for temperature and salinity were used. Here the heat and freshwater fluxes took the following forms:

$$
\begin{aligned}
& H_{\text {flux }}=\rho_{0} C_{p} H_{m} \frac{\mathrm{SST}_{\text {fix }}-\mathrm{SST}}{\tau_{T}} \text { and } \\
& F_{\text {flux }}=H_{m} \frac{\mathrm{SSS}_{\mathrm{fix}}-\mathrm{SSS}}{\tau_{S}}
\end{aligned}
$$

where $\tau_{T}$ and $\tau_{S}$ are the restoring time scales for SST and SSS, respectively; $\rho_{0}$ is the reference density; $C_{p}$ is the specific heat at constant pressure; and $H_{m}$ is the depth of the surface layer. The quantities $\mathrm{SST}_{\text {fix }}$ and $\mathrm{SSS}_{\text {fix }}$ are the prescribed restoring SST and SSS in the forms of

$$
\begin{aligned}
& \operatorname{SST}_{\text {fix }}=A_{T} \cos \left(\frac{\phi-\phi_{S}}{\phi_{N}-\phi_{S}}\right) \quad \text { and } \\
& \operatorname{SSS}_{\text {fix }}=A_{S} \cos \left(\frac{\phi-\phi_{S}}{\phi_{N}-\phi_{S}}\right)
\end{aligned}
$$

where $A_{T}$ and $A_{S}$ are the amplitudes of $\mathrm{SST}_{\mathrm{fix}}$ and $\mathrm{SSS}_{\mathrm{fix}}$, respectively, and $\phi$ is the latitude. In addition, $\phi_{S}$ and $\phi_{N}$ are the latitudes of the southern and northern boundaries $\left(10^{\circ}\right.$ and $\left.74^{\circ} \mathrm{N}\right)$, respectively.

Convective processes are unresolved because of the coarse resolution of the model, and hence a convection parameterization scheme, in the form of local implicit mixing, was used (Dijkstra et al. 2001). In this scheme the vertical tracer diffusive coefficient is enlarged by a factor of 250 , if the fluid column becomes unstably stratified. The representation of the vertical convection parameterization in the temperature $T$ and salinity $S$ equations can be written as

$$
C=\frac{\partial}{\partial z}\left[K_{V}^{C} F\left(N_{b}^{2}\right) \frac{\partial \mu}{\partial z}\right],
$$

where $K_{V}^{C}$ is the vertical convective coefficient (Table 1 ) and $\mu$ is either $T$ or $S$. In addition, $N_{b}=\sqrt{-g \rho_{0}^{-1} \partial \rho / \partial z}$ is the buoyancy frequency and $F$ is a smoothing operator of the form

$$
F(x)=\max \left[\tanh \left(-x^{3}\right), 0\right],
$$

which is needed for the Newton-Raphson method in THCM to converge (de Niet et al. 2007).

\section{b. Basic state}

Once the steady state was reached by direct time integration from the motionless state, the heat and freshwater fluxes $H_{\text {flux }}$ and $F_{\text {flux }}$ were diagnosed and used subsequently in prescribed flux boundary conditions. An integration for 1000 years starting from this steady state confirmed that it is indeed maintained by the prescribed time-independent flux boundary conditions.

The properties of the basic steady state are presented in Fig. 1. The meridional overturning stream function $\psi$, defined by $\bar{v}^{\theta}=-\partial \psi / \partial z$ and $\bar{w}^{\theta}=\partial \psi / R_{0} \partial \phi$, where $R_{0}$ is the radius of Earth and $\bar{v}^{\theta}$ and $\bar{w}^{\theta}$ are the zonal integrals of meridional and vertical velocities, respectively, measures the total meridional volume flux across a zonal section integrated from the bottom to a specific depth and thus presents the strength of the meridional overturning circulation (MOC). As shown in Fig. 1a, the current flows northward in the upper level, sinks in a narrow zone $\left[64^{\circ} \mathrm{N}\right.$, $74^{\circ} \mathrm{N}$ ], spreads as North Atlantic Deep Water, and finally returns to the upper level by mixing and upwelling processes. The maximum of the meridional overturning stream function (hereinafter MMSF) is about $16 \mathrm{~Sv}$ (located at a depth of $1500 \mathrm{~m}$ ) and in the right range compared to observed values (Cunningham et al. 2007; Hobbs and Willis 2012). The zonal overturning stream function $\chi$, defined by $\bar{u}^{\phi}=-\partial \chi / \partial z$ and $\bar{w}^{\phi}=\left(R_{0} \cos \phi\right)^{-1} \partial \chi / \partial \theta$, where $\bar{u}^{\phi}$ and $\bar{w}^{\phi}$ are meridional integrals of zonal and vertical velocities, respectively, shows the strength of zonal overturning circulation (ZOC). Figure $1 \mathrm{~b}$ shows that an eastward current flows close to the surface, sinks near the eastern boundary, then flows westward, and finally upwells near the western boundary. The zonal mean density distribution (Fig. 1c) shows that the isopycnals gradually rise 

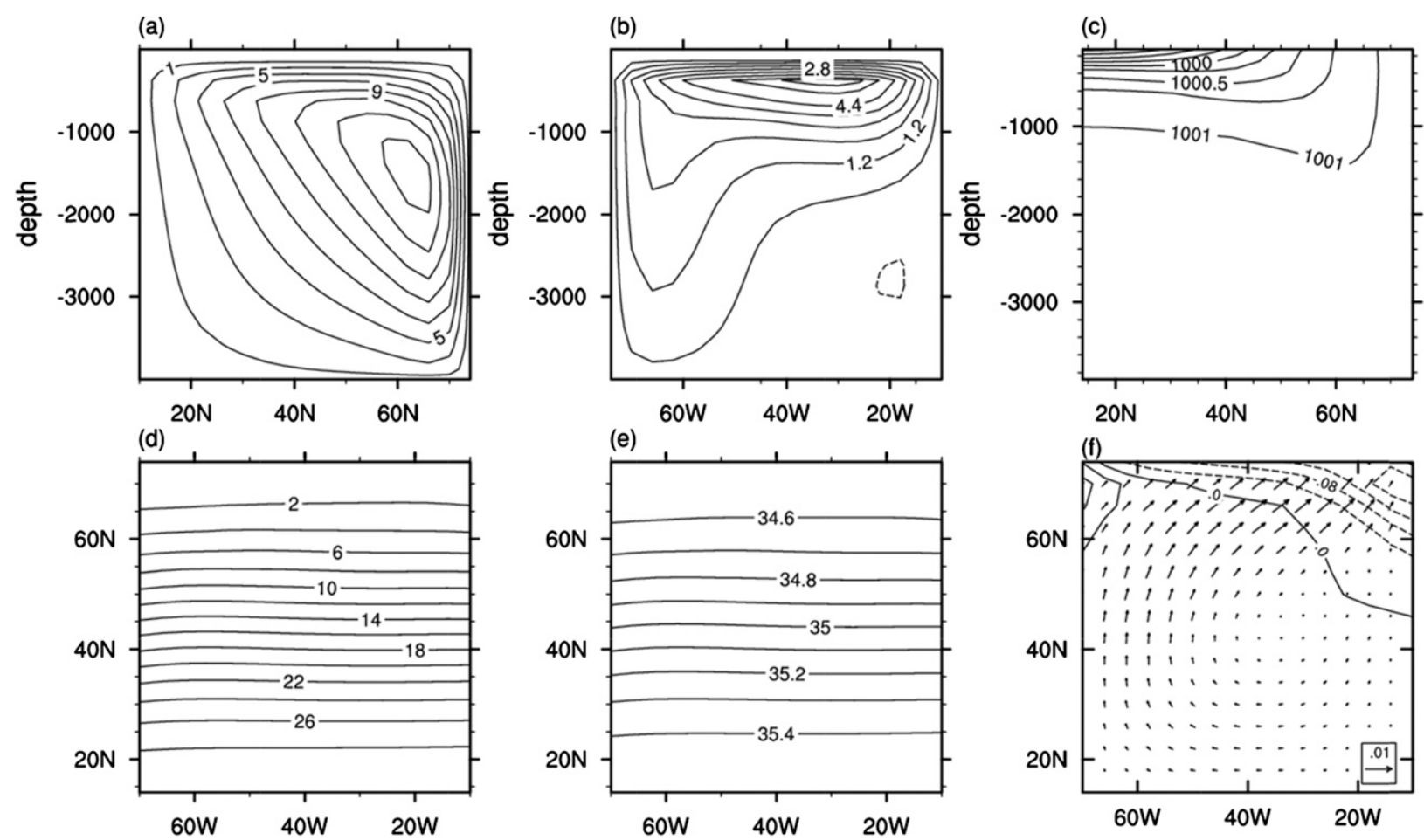

FIG. 1. Properties of the basic state, with the (a) meridional and (b) zonal streamfunctions (Sv), (c) zonal mean density $\left(\mathrm{kg} \mathrm{m}^{-3}\right),(\mathrm{d}) \mathrm{SST}\left({ }^{\circ} \mathrm{C}\right)$, (e) SSS (psu), and (f) the velocity averaged from the surface to $1500 \mathrm{~m}_{\text {depth }}\left(\mathrm{m} \mathrm{s}^{-1}\right)$. The solid and dashed lines represent the positive and negative values, respectively, in (b) and (f), and the lines and arrows denote the vertical and horizontal velocities, respectively, in (f).

toward high latitudes, indicating the cooling at high latitudes that is associated with strong convection. The SST (Fig. 1d) and SSS (Fig. 1e) present nearly zonally uniform pattern, with the range of $2^{\circ}-28^{\circ} \mathrm{C}$ and $34.6-$ $35.4 \mathrm{psu}$, respectively. The horizontal and vertical velocities averaged over the upper levels (from the surface to $1500-\mathrm{m}$ depth) show that a current flows from the southwestern part to the northeastern part and then sinks near the northern boundary, mostly concentrating in the northeastern corner (Fig. 1f). The depthmean current below $1500 \mathrm{~m}$ is similar, but with opposite direction (not shown). This steady state is reasonable in view of the idealized configuration and was used as the basic state below.

\section{c. The method}

To calculate the NOIP, the conditional nonlinear optimal perturbation (CNOP) method was used $(\mathrm{Mu}$ et al. 2003). We write the THCM model equations as

$$
\left\{\begin{array}{l}
\partial \mathbf{X} / \partial t=F(\mathbf{X}) \\
\left.\mathbf{X}\right|_{t=0}=\mathbf{X}_{0}
\end{array}\right.
$$

where $\mathbf{X}$ is the state vector and $\mathbf{X}_{0}$ is the initial condition. The solution can be written formally as

$$
\mathbf{X}(\tau)=M_{0, \tau}\left(\mathbf{X}_{0}\right),
$$

where $\mathbf{X}(\tau)$ is the solution at the time $\tau$, and $M_{0, \tau}$ is the nonlinear propagation operator that corresponds to the integration of (7) from the initial time $t=0$ to $t=\tau$. Therefore, for an initial condition $\mathbf{U}_{0}$ and for one with a small perturbation $\mathbf{U}_{0}+\mathbf{u}_{0}$, the solutions can be written as

$$
\left\{\begin{array}{l}
\mathbf{U}(\tau)=M_{0, \tau}\left(\mathbf{U}_{0}\right) \\
\mathbf{U}(\tau)+\mathbf{u}(\tau)=M_{0, \tau}\left(\mathbf{U}_{0}+\mathbf{u}_{0}\right)
\end{array}\right.
$$

where $\mathbf{u}(\tau)$ is called the nonlinear evolution of the initial perturbation $\mathbf{u}_{0}$ with respect to $\mathbf{U}(\tau)$, representing the influence of $\mathbf{u}_{0}$ on $\mathbf{U}(\tau)$. Suppose that the initial perturbation $\mathbf{u}_{0}$ satisfies a given constraint condition $\mathbf{u}_{0} \in C_{\delta}$, then the initial perturbation $\mathbf{u}_{0}^{*}$ is called the CNOP, if and only if

$$
J\left(\mathbf{u}_{0}^{*}\right)=\max _{u_{0} \in C_{\delta}} J\left(\mathbf{u}_{0}\right),
$$

where $J$ is called the objective function, given by

$$
J\left(\mathbf{u}_{0}\right)=\left\|M_{0, \tau}\left(\mathbf{U}_{0}+\mathbf{u}_{0}\right)-M_{0, \tau}\left(\mathbf{U}_{0}\right)\right\| .
$$

It measures the change of $\mathbf{u}(\tau)$ caused by $\mathbf{u}_{0}$ in (9) under a given norm $\|\cdot\|$. From the definition, it is obvious 
that the CNOP is the initial perturbation, which can develop fastest in the nonlinear regime and therefore induces the largest modification (measured by $J$ ) of the state vector $\mathbf{U}(\tau)$ at the time $\tau$, with respect to other perturbations satisfying $\mathbf{u}_{0} \in C_{\delta}$.

The CNOP method yields the fastest-growing perturbation in fully nonlinear models, and it can thus be used to study the stability and predictability of atmosphere and ocean flows (Mu et al. 2006; Duan and $\mathrm{Mu}$ 2009). In the past few years, the CNOP method has been used in the study of the predictability of ENSO events (Duan and Mu 2006; Mu et al. 2007), the Kuroshio large meander variability (Wang et al. 2012, 2013), the sensitivity of ecosystems (Sun and Mu 2011), the sensitivity and multidecadal variability of AMOC (Mu et al. 2004; $\mathrm{Zu}$ et al. 2013), and to determine adaptive observation strategies of typhoons (Qin and Mu 2012).

The first singular vector (FSV) method produces the fastest-growing initial perturbations in the tangent linear models (Farrell and Ioannou 1996). Given the norm $N$ and the optimization time $\tau$, the FSV can be obtained by estimating the leading eigenvector of $R_{0, \tau}^{\dagger} N R_{0, \tau}$, where $R_{0, \tau}$ is the tangent linear propagator and $R_{0, \tau}^{\dagger}$ its adjoint. In fact, the FSV can be considered as a linear combination of the eigenvectors of $R_{0, \tau}$, that is, $S^{v}=\sum_{i} \sigma_{i} E_{i}$, where $S^{v}$ is the FSV,$E_{i}$ is an eigenvector of $R_{0, \tau}$, and $\sigma_{i}$ is the amplitude of the $E_{i}$. In the linear transformation $R_{0, \tau} S^{v}, E_{i}$ will stretch (positive eigenvalue) or squeeze (negative eigenvalue) independently, that is, $R_{0, \tau} S^{v}=\sum_{i} \sigma_{i}\left(R_{0, \tau} E_{i}\right)$. Usually, the tangent linear operator of the ocean model is nonnormal $\left(R_{0, \tau}^{\dagger} R_{0, \tau} \neq\right.$ $R_{0, \tau} R_{0, \tau}^{\dagger}$ ) because of advection and convection, such that in the linear combination $S^{v}=\sum_{i} \sigma_{i} E_{i}$, some $E_{i}$ are possibly not pairwise orthogonal. In this case, the amplitude of the first eigenvector $E_{1}$, that is, $\sigma_{1}$, can be relatively large, even larger than one. The $S^{v}$ can develop fastest in the tangent linear model, because $E_{1}$ has a large initial amplitude, and at the same time $E_{1}$ develops fastest (Tziperman and Ioannou 2002).

The CNOP method is an extension of the FSV method to the nonlinear regime (Kerswell et al. 2014). A CNOP is theoretically defined as the fastest growing perturbations of all the possible ones in the fully nonlinear model. It is practically obtained by an optimization algorithm, here the Spectral Projected Gradient 2 (SPG2) method (Birgin et al. 2000). The growth of the CNOP in a nonlinear model possibly involves both nonnormal interactions of the eigenvectors, like the FSV, and nonlinear interactions. In addition, for the given optimization time $\tau$, constraints $C_{\delta}$, and objective function $J$, there exists a first singular vector, as well as a second, a third, etc. When the nonlinear evolution is considered, for the given $\tau, C_{\delta}$, and $J$, the maxima of $J$ can be caused by several initial perturbations, of which the ones causing the global maximum are called CNOP and the ones causing local maxima are called local CNOPs.

In this study, the perturbations of FSV type were also computed. It should be noted that, according to the definition, the pattern of the FSV-type perturbations is independent of the perturbation amplitude. However, in the nonlinear regime, the contributions of nonlinear terms are not proportional to the amplitude of initial perturbations, and the pattern of the CNOP-type perturbations is dependent on the amplitude ( $\mathrm{Mu}$ et al. 2009). Therefore, to compare these two kinds of perturbations, the optimal initial perturbations of FSV type will be calculated under relatively small amplitude and then rescaled to the amplitude of the CNOP.

\section{d. Optimization configurations}

The steady state in section $2 \mathrm{~b}$ was chosen to be the time-independent basic state that corresponded to the solution of $\mathbf{U}(\tau)=M_{0, \tau}\left(\mathbf{U}_{0}\right)$ in (9). As temperature and salinity anomalies are predominantly generated at the surface, only the optimal initial anomalies of SST and SSS [that correspond to $\mathbf{u}_{0}^{*}$ in (10)] were studied in this paper. The optimization time $\tau$ in (11) was chosen to be 10 years, which is long enough to cause a strong deviation in the AMOC.

The initial SST and SSS anomalies were optimized separately and the constraint condition that corresponds to $\mathbf{u}_{0} \in C_{\delta}$ in (10) takes the form of either

$$
\sqrt{\sum_{i, j \in \Lambda}\left[\mathrm{SST}_{0}^{\prime}(i, j)\right]^{2} \cos \phi_{j}} \leq \delta_{\mathrm{SST}}
$$

or

$$
\sqrt{\sum_{i, j \in \Lambda}\left[\mathrm{SSS}_{0}^{\prime}(i, j)\right]^{2} \cos \phi_{j}} \leq \delta_{\mathrm{SSS}}
$$

where $i$ and $j$ denote the grid indices in the zonal and meridional directions, which cover the sea surface $\Lambda$ in the whole model domain; $\operatorname{SST}_{0}^{\prime}(i, j)$ and $\operatorname{SSS}_{0}^{\prime}(i, j)$ are the initial anomalies of SST and SSS, respectively; and $\delta_{\mathrm{SST}}$ and $\delta_{\mathrm{SSS}}$ are called constraint radii. Given the values of $\delta_{\mathrm{SST}}$ and $\delta_{\mathrm{SSS}}$ in (12) and (13), we determined the CNOPs by solving the optimization problem (10), with the global optimum being caused by the NOIP. When the amplitudes (absolute maxima) of the SST and SSS perturbations were too large or too small, with respect to $3^{\circ} \mathrm{C}$ and 0.5 psu that are the realistic variations of observed SST and SSS (Kennedy et al. 2011; Belkin et al. 1998), the values of $\delta_{\mathrm{SST}}$ and $\delta_{\mathrm{SSS}}$ were adjusted. This process was repeated several times, and it was found that when $\delta_{\mathrm{SST}}=18^{\circ} \mathrm{C}$ and $\delta_{\mathrm{SSS}}=3.2 \mathrm{psu}$, the amplitudes of the SST and SSS perturbations were $3^{\circ} \mathrm{C}$ 
and $0.5 \mathrm{psu}$, respectively. It should be noted that the NOIPs obtained are always located on the boundaries of the constraints, that is, $\sqrt{\sum_{i, j \in \Lambda}\left[\mathrm{SST}_{0}^{\prime}(i, j)\right]^{2} \cos \phi_{j}}=\delta_{\mathrm{SST}}$ and $\sqrt{\sum_{i, j \in \Lambda}\left[\operatorname{SSS}_{0}^{\prime}(i, j)\right]^{2} \cos \phi_{j}}=\delta_{\text {Sss }}$ in our case, although the constraints allow $\sqrt{\sum_{i, j \in \Lambda}\left[\operatorname{SST}_{0}^{\prime}(i, j)\right]^{2} \cos \phi_{j}}<\delta_{\mathrm{SST}}$ and $\sqrt{\sum_{i, j \in \Lambda}\left[\mathrm{SSS}_{0}^{\prime}(i, j)\right]^{2} \cos \phi_{j}}<\delta_{\text {Sss }}$. It suggests that the NOIP with larger amplitude will develop faster.

The MMSF was used to measure the strength of the AMOC. However, the amplitude of the initial anomalies here was large enough to shift the location of the MMSF at the optimization time $\tau$ from that in the basic state. As the change of MMSF location would increase the difficulty during optimization, a slightly different objective function was used to measure the variations of the AMOC strength, that is,

$J(\tau)=\frac{1}{2} \int_{\text {South }}^{\text {North }}\left[\frac{R_{0}}{r} \int_{\text {West }}^{\text {East }} \int_{L}^{\text {Surface }} v_{\tau}^{\prime} \cos \phi d \theta d z\right]^{2} d \phi$,

where $v_{\tau}^{\prime}$ is the meridional velocity anomaly at the optimization time $\tau$; North, South, East, West, and Surface are the lateral and surface boundaries of the model, respectively; $\phi, \theta$, and $z$ are the latitude, longitude, and depth, respectively; $L$ is the MMSF depth $(1500 \mathrm{~m})$ in the basic state; $R_{0}$ is again the radius of Earth $(6.37 \times$ $\left.10^{6} \mathrm{~m}\right)$; and $r=10^{6}$ is used to rescale $J$ to a relatively small value for the convenience of the computation of the CNOP. The objective function measures the integral change of the meridional volume flux in the upper level (Alexander and Monahan 2009), and its use avoids the problem caused by the change of MMSF location during optimization.

\section{The NOIP and the resulting AMV}

\section{a. The NOIPs of SSS and SST}

The NOIP of SSS is characterized by a strong meridional gradient and a weak zonal one, with the absolute maximum in the northwestern part of the basin (Fig. 2a). The pattern is consistent with the LOIP of SSS in Sévellec et al. (2009) under prescribed heat or freshwater flux boundary conditions. The negative values (fresher anomalies) are located in the north part, which is in agreement with the fact that the AMOC is more sensitive to the anomalies of freshwater forcing at high latitudes (Manabe and Stouffer 1997). Moreover, the absolute maximum is $0.5 \mathrm{psu}$, in agreement with the typical amplitude of SSS anomalies in the GSA events (Belkin et al. 1998). The NOIP of SST (Fig. 2b) shows a similar pattern as SSS, with a strong (weak) meridional (zonal) gradient and the absolute maximum in the northwestern part of the basin. Moreover, the absolute maximum of SST anomalies is around $3^{\circ} \mathrm{C}$, of the same amplitude as strong seasonal SST anomalies in the HadSST dataset (Kennedy et al. 2011).

$\mathrm{Zu}$ et al. (2013) calculated the NOIP of SSS under mixed boundary conditions. Because of the atmospheric damping of SST anomalies, the NOIP of SSS has different characteristics: the negative anomalies in the northwest are weaker, whereas the positive anomalies become stronger and move to the western subbasin near $40^{\circ} \mathrm{N}$ (with respect to the results under flux boundary conditions). These differences are also found in Sévellec et al. (2009), which indicate that the pattern of optimal initial anomalies depends on the type of the surface boundary conditions for SST and SSS.

The NOIPs of SSS and SST correspond to similardensity anomaly patterns, with the largest signal in the northwestern part of the basin. Both positive SST anomalies and negative SSS anomalies lead to negative density anomalies, which dominate the evolution of the velocity field. This indicates that the AMOC will likely change the most after 10 years if the corresponding anomalies (negative salinity ones or positive temperature ones) occur in the northwestern part of the basin. From the view of adaptive observations (Duan et al. 2004), the pattern of the anomalies suggests that the forecast skill on a decadal time scale can be improved the most, if a limited observation system is deployed in the northwestern part of the basin to monitor the thermohaline changes.

\section{b. The AMV caused by the NOIP}

The NOIP of SSS and SST were used to initialize the nonlinear model, and the resulting AMV is investigated in this section. The MMSF and the objective function $J$ in (14) are used to represent the MOC strength from a local and integral view, respectively.

Figures $2 \mathrm{c}$ and $2 \mathrm{~d}$ show that both the NOIPs of SSS and SST can induce multidecadal oscillations of the AMOC. The period of the oscillations is around 50 years, which is in accordance with the observed variability (Enfield et al. 2001). The velocity anomalies, as represented by the MMSF and the objective function $J$, gradually decay with time and finally return to the basic state (not shown). It suggests that the SSS and SST perturbations with realistic amplitudes, no matter what the patterns are, cannot induce an abrupt change of the AMOC, like a shift between different steady states. In other words, the AMOC is stable in the nonlinear framework using this model.

After superimposing the NOIP of SSS, the MMSF increases in the first 2 years and then decreases substantially (Fig. 2c). After 10 years the MMSF reaches the 
(a)

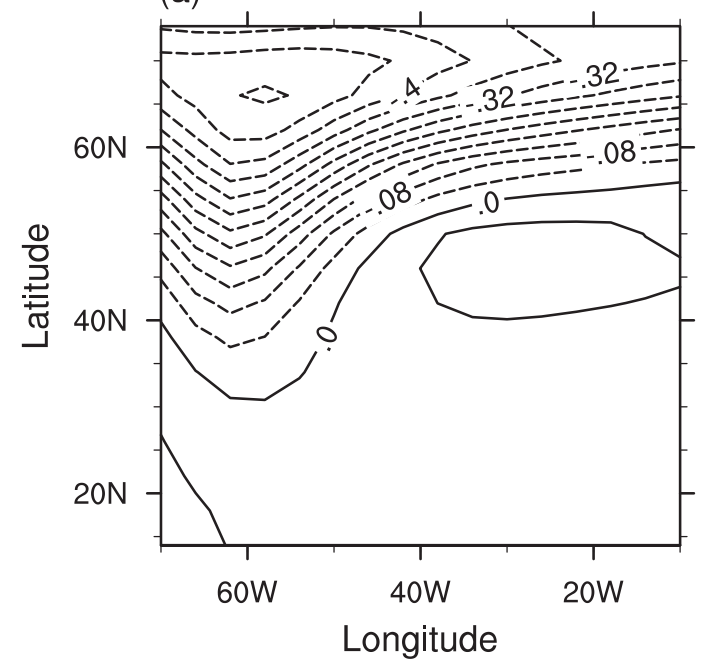

(c)

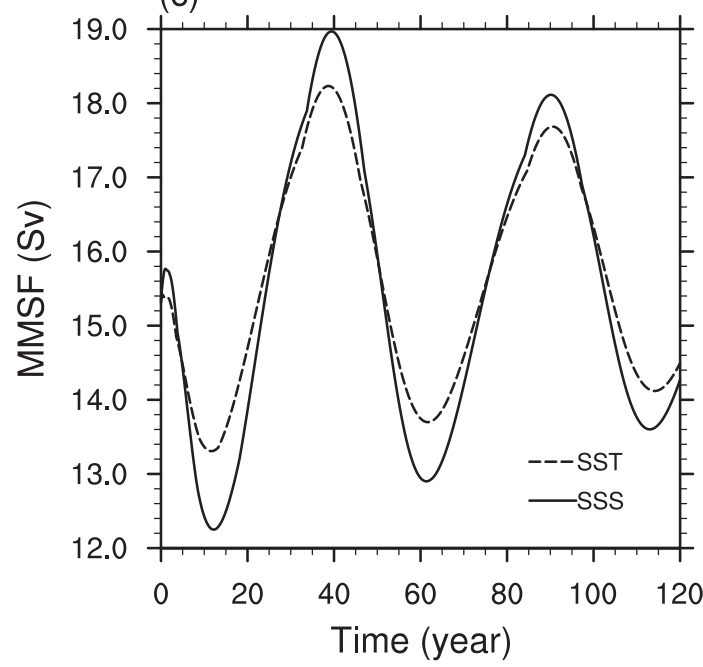

(b)

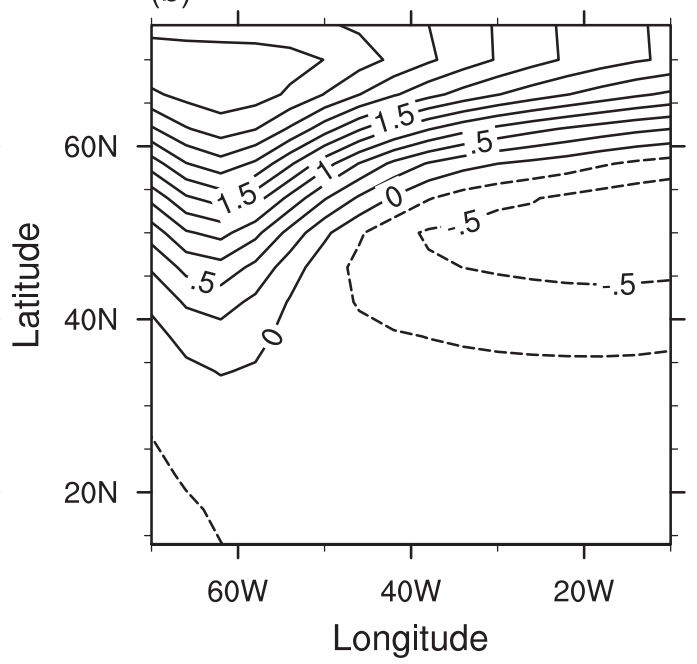

(d)

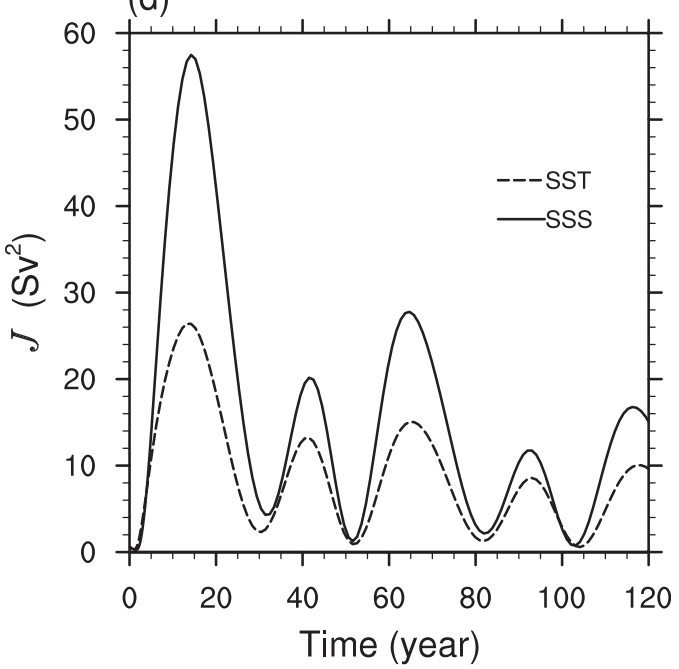

FIG. 2. The NOIP (i.e., CNOP) of (a) SSS (psu) and (b) SST (C), under the constraints $\delta_{\mathrm{SsS}}=3.2$ psu and $\delta_{\mathrm{SST}}=$ $18^{\circ} \mathrm{C}$ and an optimization time of 10 years; the resulting multidecadal oscillations, measured by (c) MMSF and (d) the objective function $J$, in the nonlinear model.

minimum of $12.25 \mathrm{~Sv}$, which is a reduction of $3.6 \mathrm{~Sv}$ $(22.7 \%)$ of that of the basic state. The slowdown here is stronger than the results of Sévellec et al. (2009); the latter is only $2.25 \mathrm{~Sv}$ for the LOIP of SSS with the same amplitude. For the SST anomalies, the resulting oscillation is weaker than the case of SSS anomalies (Fig. 2c). After 10 years the MMSF reaches the minimum of $13.31 \mathrm{~Sv}$, that is, a reduction of $2.54 \mathrm{~Sv}(15.8 \%)$ of that of the basic state.

The objective function (Fig. 2d) also clearly shows this difference from an integral point of view: the variations of $J$ by the SSS anomalies are twice as large as those by SST anomalies during the peak time ( $t=15$ years). The results here indicate that although it is difficult to distinguish the respective contributions of SST and SSS to the observed multidecadal variability (Wang et al. 2010), SSS anomalies give a much larger response in the AMOC than SST anomalies.

\section{c. NOIP of SSS with different optimization time}

Both the LOIP and NOIP are dependent on the optimization time, that is, one obtains different patterns under different optimization time. Regarding targeted observations, it suggests that different areas should be monitored for the forecasts with different lead time. When using the LOIP, often the optimal time, that is, the optimization time at $t=\tau$ that corresponds to the maximum of the objective function is determined (Sévellec et al. 2009; Zanna et al. 2012). The optimal time indicates "the optimal pattern" in the time dimension. 
(a)
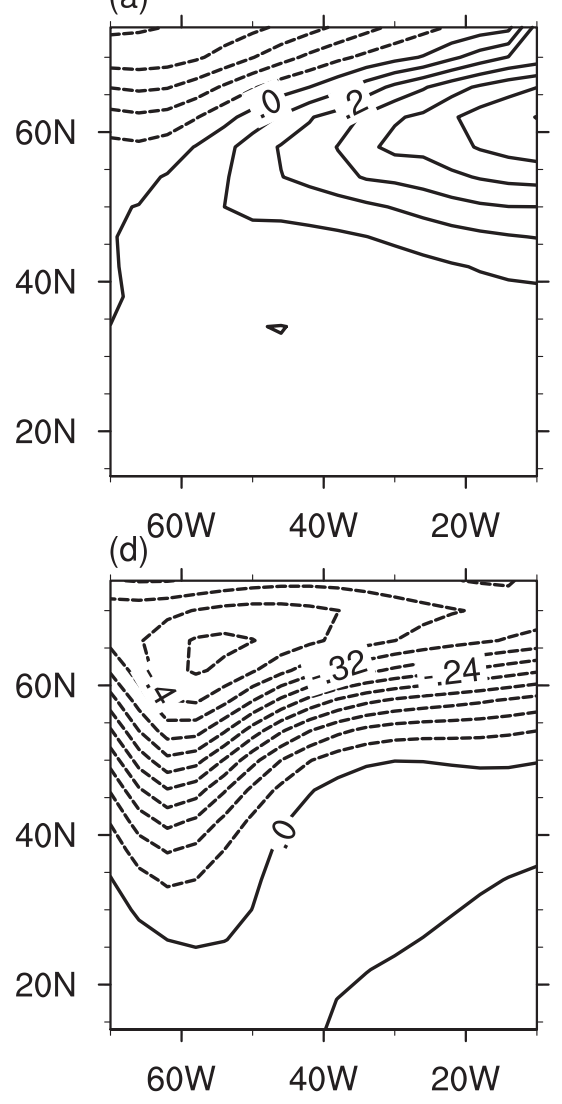

(b)

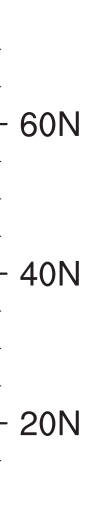

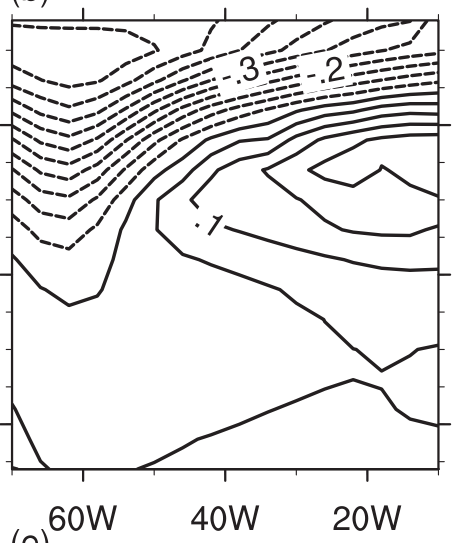

(e)

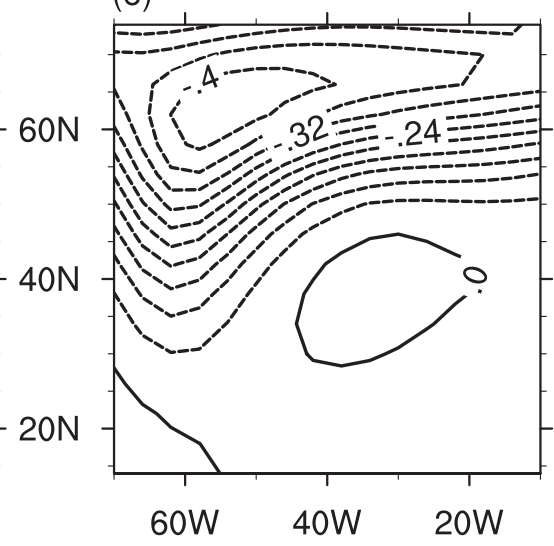

(c)

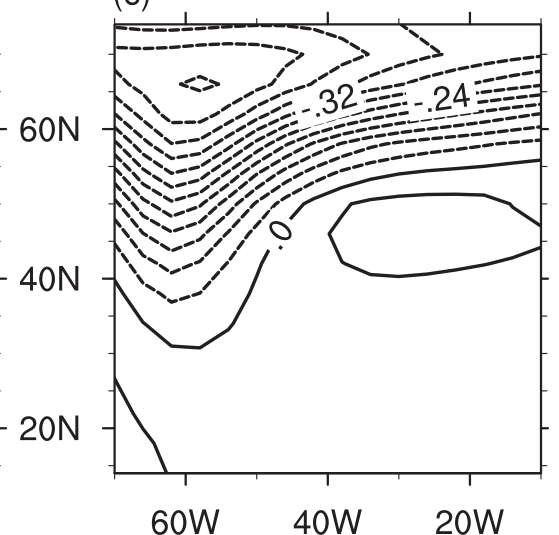

(f)

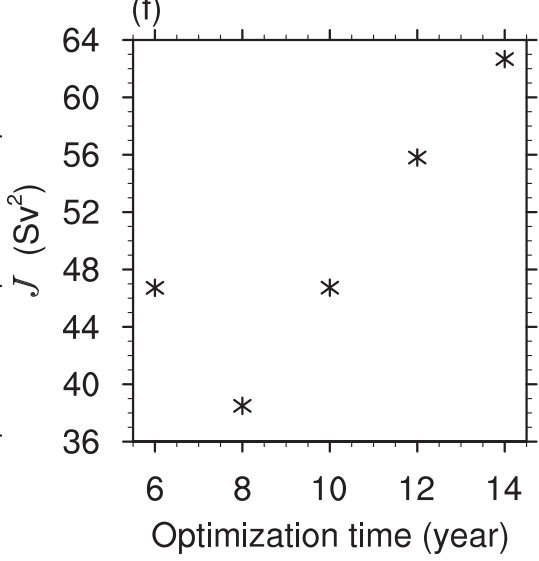

FIG. 3. Spatial patterns of the NOIP of SSS with an optimization time of (a) 6, (b) 8, (c) 10, (d) 12, and (e) 14 years, and (f) the resulting objective function values. The constraint radii are all set $\delta_{\mathrm{Sss}}=3.2 \mathrm{psu}$.

The calculation of the CNOP involves integrating the nonlinear and adjoint models repeatedly and is therefore computationally intensive. Limited by the computation of CNOP, we only optimize five cases with the optimization time of $6,8,10,12$, and 14 years, respectively. As shown in Figs. 3a-e, the NOIPs of SSS are dependent on the optimization time. With the extension of the optimization time, the anomalies move gradually eastward. This indicates that for a different lead time, different key areas should be monitored. Specifically, the eastern boundary near $60^{\circ} \mathrm{N}$ is the key area when the optimization time is 6 years, while the northwestern part (the region $\left[40^{\circ} \mathrm{W}, 60^{\circ} \mathrm{W}\right] \times\left[60^{\circ} \mathrm{N}, 70^{\circ} \mathrm{N}\right]$ ) becomes a key area when the optimization time is 15 years. The resulting objective function $J$ is minimal for 8 years, and $J$ increases for both smaller and larger optimization times (Fig. 3f).

\section{The physics of the excitation of the AMV}

It is found that the AMV induced by the NOIP of SST or SSS is similar: both kinds of perturbations lead to similar density anomalies and AMV through similar dynamics. However, the AMV excited by the NOIP of SST is relatively weak, and therefore we only consider the case where it is excited by the NOIP of SSS.

\section{a. The basic mechanism of the AMV in THCM}

According to previous studies (e.g., Dijkstra et al. 2006), the propagation of the pattern of the AMV is mainly dominated by linear physics, involving the interaction of zonal and meridional density gradient and the velocities (thermal wind balance). Here this mechanism is employed to explain the oscillation excited by the NOIP of SSS during the slow-evolution period (years 30-120).

To show the phase relationship of the density gradient and velocities, several indices were calculated during years $0-120$. First, the anomalies of MMSF and the maximum of zonal overturning stream function with respect to the basic state were calculated and denoted by MOC' and $\mathrm{ZOC}^{\prime}$, respectively. For simplification, the zonal (meridional) density gradient was approximated by the density difference between the eastern and western (northern and 


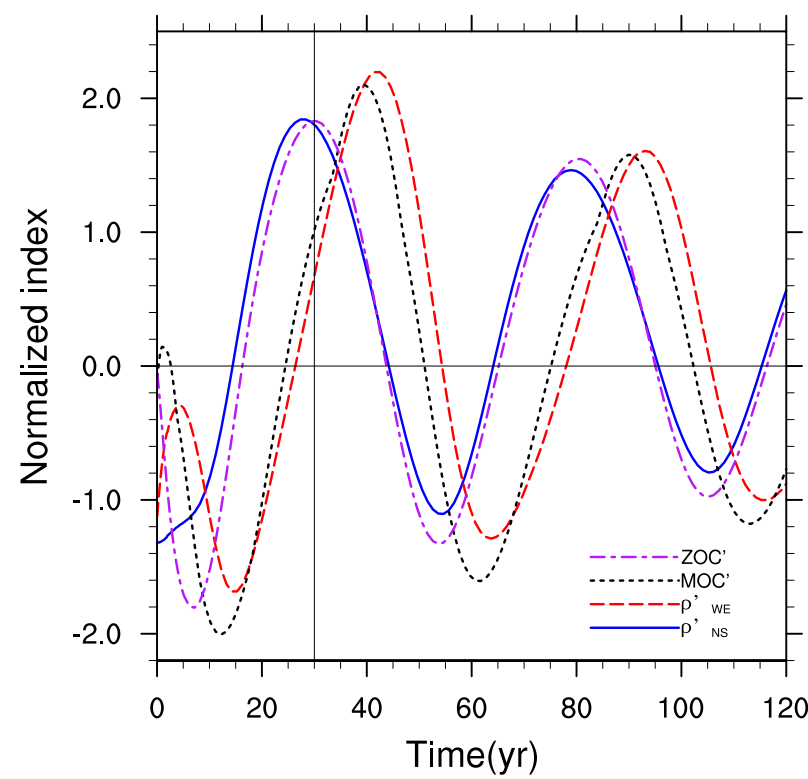

FIG. 4. The normalized time series of $\mathrm{ZOC}^{\prime}, \mathrm{MOC}^{\prime}, \rho_{W E}^{\prime}$, and $\rho_{N S}^{\prime}$.

southern) equally divided subbasins and denoted by $\rho_{E W}$ $\left(\rho_{N S}\right)$. The difference between $\rho_{E W}\left(\rho_{N S}\right)$ and that of the basic state was denoted by $\rho_{E W}^{\prime}\left(\rho_{N S}^{\prime}\right)$. It should be noted that the opposite of the zonal density difference, $\rho_{W E}^{\prime}$, was used to show clearly its phase relationship with $\mathrm{MOC}^{\prime}$. Finally, the four indices $\left(\mathrm{MOC}^{\prime}, \mathrm{ZOC}^{\prime}, \rho_{W E}^{\prime}\right.$, and $\left.\rho_{N S}^{\prime}\right)$ were normalized during years $0-120$, according to

$$
\tilde{\beta}=\frac{\beta-\operatorname{mean}(\beta)}{\operatorname{std}(\beta)},
$$

where $\beta$ denotes the indices and mean $(\beta)$ and $\operatorname{std}(\beta)$ are the mean value and standard deviation of $\beta$, respectively.

During the first 30 years, the four indices are out of phase, which corresponds to a fast adjustment, and nonlinear processes likely play an important role (Fig. 4). After 30 years, the MOC' and $\rho_{W E}^{\prime}\left(\mathrm{ZOC}^{\prime}\right.$ and $\left.\rho_{N S}^{\prime}\right)$ have a fixed phase relation, suggesting that linear mechanisms become a dominating factor. Specifically, at year 30 (solid vertical line in Fig. 4), $\rho_{N S}^{\prime}$ shows a positive anomaly with respect to the basic state. A $\rho_{N S}^{\prime}>0$ leads to positive $\mathrm{ZOC}^{\prime}$ owing to the thermal wind relation during years $30-40$. Moreover, $\mathrm{ZOC}^{\prime}>0$ causes the upwelling (downwelling) anomaly in the western (eastern) subbasin, with respect to the basic state, which in turn increases (decreases) the density in the western (eastern) subbasin. Therefore, during years 30-40, $\rho_{W E}^{\prime}$ increases and the $\mathrm{MOC}^{\prime}$ also increases correspondingly due to the thermal wind relation. On the other hand, MOC $^{\prime}>0$ means that there exists an upwelling (downwelling) anomaly in the southern (northern) subbasin. Hence, a MOC' $>0$ will cause the decrease of
$\rho_{N S}^{\prime}$, and $\mathrm{ZOC}^{\prime}$ also decreases by the thermal wind relation. These interactions compose the $1 / 4$ phase of the oscillation, until $\mathrm{ZOC}^{\prime}$ and $\rho_{N S}^{\prime}$ reach zero and $\mathrm{MOC}^{\prime}$ and $\rho_{W E}^{\prime}$ reach the maximum approximately at year 40 . At other phases, the processes are similar: the velocity anomaly influences the density gradient anomaly through advection and convection, while the density gradient anomaly, in turn, induces a velocity anomaly by the thermal wind relation. In te Raa and Dijkstra (2002), this mechanism was proposed to explain the propagation features of the AMOC multidecadal oscillation, and the characteristics of this mechanism were also found in Sévellec et al. (2009). It should be noted that the spatial pattern was also referred to as the "thermal Rossby mode" (Colin de Verdière and Huck 1999; Huck and Vallis 2001; Dijkstra 2013).

\section{b. The effects of nonlinear processes}

As mentioned above, the velocity anomalies do not correlate with the anomalies of the density gradient during years $0-30$, which indicates that the nonlinear processes likely exert an important influence on the evolution of the initial anomalies. In this section, the contributions of the nonlinear processes during years 0-30 are analyzed.

According to the equations used in the THCM model, the nonlinear processes include the following: 1 ) in the density equation that is from the combination of $T$ and $S$ equations according to the linear equation of state used in THCM, $u^{\prime}\left(R_{0} \cos \phi\right)^{-1} \partial \rho^{\prime} / \partial \theta, v^{\prime} R_{0}^{-1} \partial \rho^{\prime} / \partial \phi, w^{\prime} \partial \rho^{\prime} / \partial z$, where the prime denotes the anomaly variables with respect to the basic state; 2) the nonlinear convection parameterization for density that is an implicit nonlinear process; and 3$)$ in the momentum equations, $u^{\prime}\left(R_{0} \cos \phi\right)^{-1} \partial u^{\prime} / \partial \theta$, $v^{\prime} R_{0}^{-1} \partial u^{\prime} / \partial \phi, w^{\prime} \partial u^{\prime} / \partial z, u^{\prime}\left(R_{0} \cos \phi\right)^{-1} \partial v^{\prime} / \partial \theta, v^{\prime} R_{0}^{-1} \partial v^{\prime} / \partial \phi$, $w^{\prime} \partial v^{\prime} / \partial z, u^{\prime} v^{\prime} \tan \theta / R_{0}$, and $-u^{\prime 2} \tan \theta / R_{0}$.

Below, we also consider the development of perturbations in partly linearized variants of the THCM, in which one of the nonlinear processes has been neglected. Especially, the nonlinearity of convection parameterization is implicit. Linearizing this process means substituting the stratification with the basic-state stratification. After linearization, these partly and also the fully linearized models were initialized by the NOIP of SSS and integrated for 120 years under the same boundary conditions. The difference of the AMV in these models, if any, should be attributed to the omitted nonlinear processes.

During years 0-30, the AMV in some of partly linearized models shows significant differences from that in the fully nonlinear model (Fig. 5). Specifically, during years $0-4$, the contribution of the nonlinear convection process is significant. Linearizing the convection 
(a)

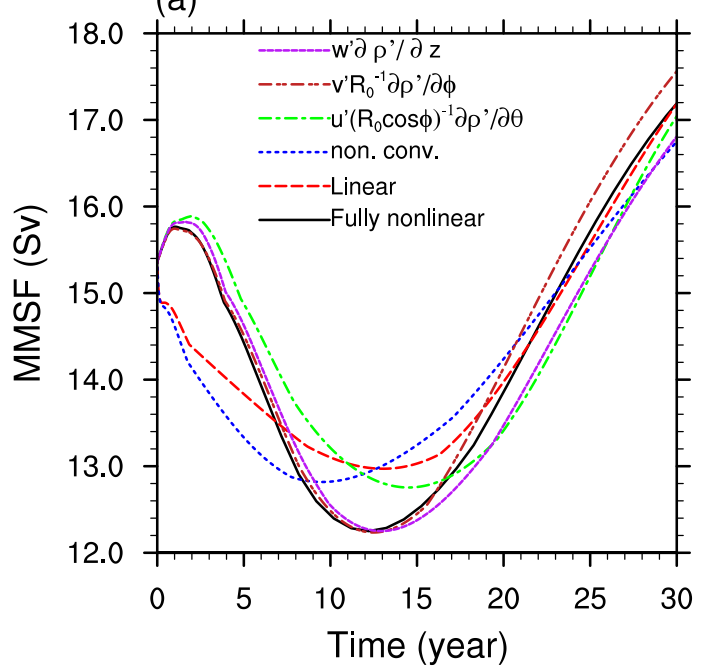

(c)

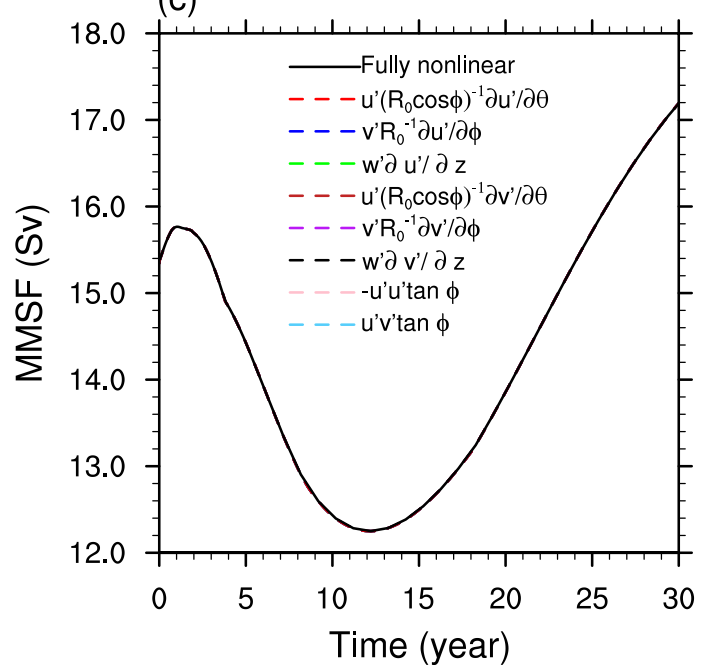

(b)

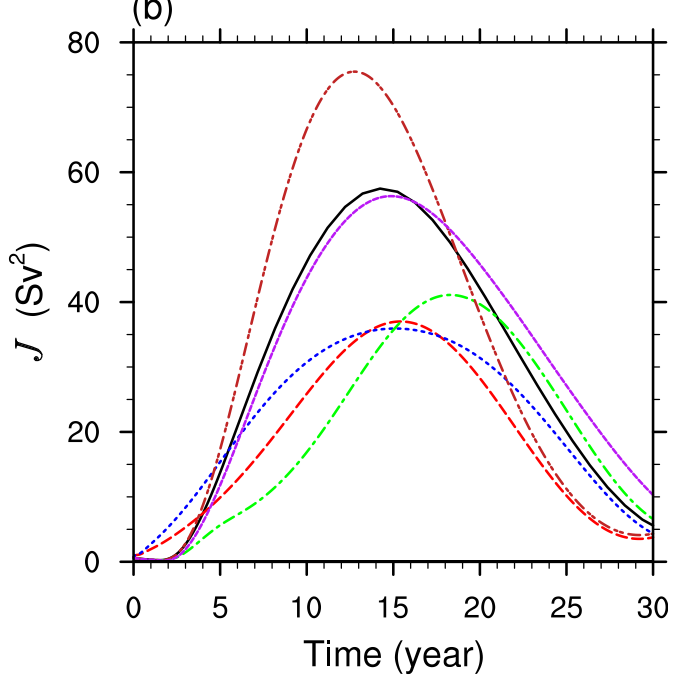

(d)

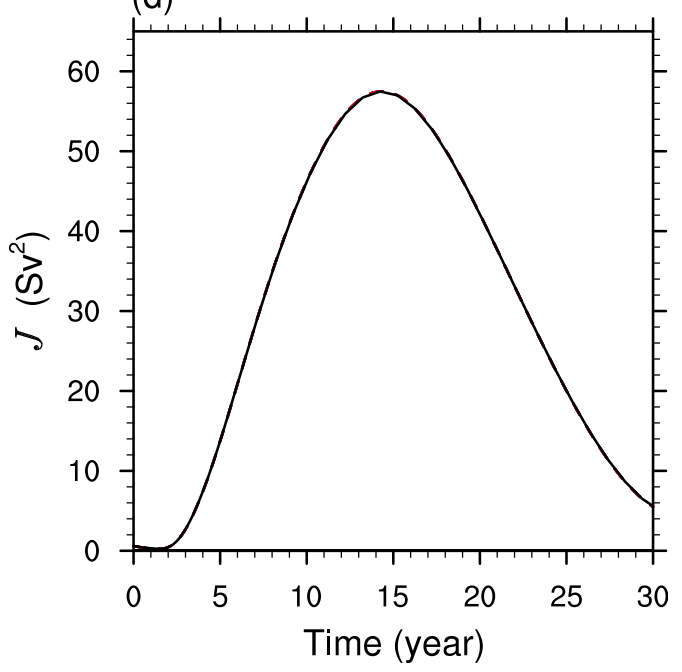

FIG. 5. The variations of (a),(c) MMSF and (b),(d) objective function $J$ in different cases caused by NOIP of SSS. "Fully nonlinear" denotes the case of the fully nonlinear model, "linear" represents the case removing all the nonlinear processes in all the equations, "non. conv." represents the case removing the nonlinear process in the convection parameterization, and the rest of terms represent the cases removing each one from the fully nonlinear model, respectively. The legend of (b) is the same as (a) and the legend of (d) is the same as (c). Note that the lines representing the nonlinear processes in the momentum equations (c) and (d) all coincide with that of the fully nonlinear case.

parameterization weakens the MOC directly (non. conv. in Fig. 5a), whereas in the fully nonlinear model, the MOC is strengthened for about 2 years. The objective function also increases directly after linearizing this process (non. conv. in Fig. 5b), indicating that the upperlevel velocities change (weaken) immediately, consistent with the behavior of the MMSF.

In fact, in the non. conv. case, the convection is dominated by the stratification of the basic state (steady state) and hence is time independent, as shown in Fig. 6a for a typical case in $70^{\circ} \mathrm{N}$. Therefore, in the non. conv. case the initial SSS anomalies are convected downward in the western subbasin, whereas they are kept more at the surface in the fully nonlinear case because of the stable stratification and the shutdown of the convection (Fig. 6b). The negative density anomalies from the surface to $300 \mathrm{~m}$ depth in the fully nonlinear case form negative pressure anomalies and excite downwelling anomalies in the northwestern corner (Fig. 6c), which then strengthen the MOC.

During years 7-20, removing the nonlinear zonal advection process $u^{\prime}\left(R_{0} \cos \phi\right)^{-1} \partial \rho^{\prime} / \partial \theta$ strengthens the MOC relatively (slow down of the weakening of the MOC, as shown in Fig. 5a). In Fig. 5b, the upper-level 
(a)

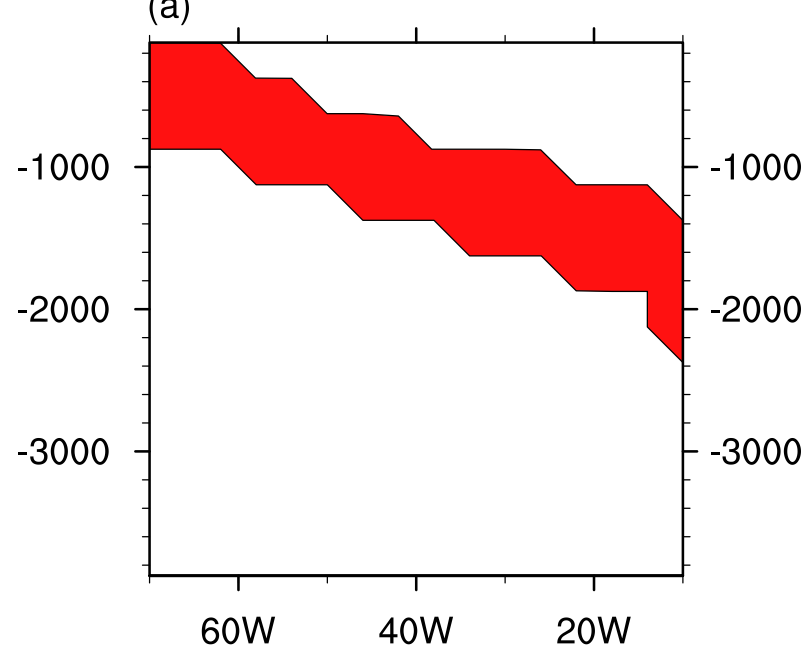

(b)

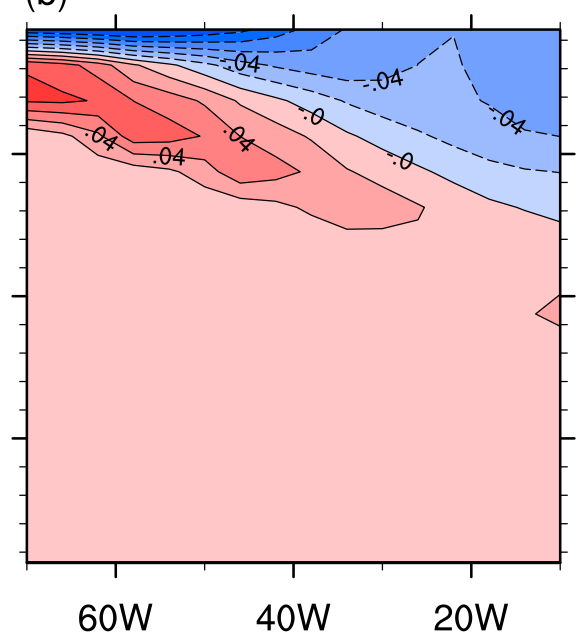

\section{(c)}

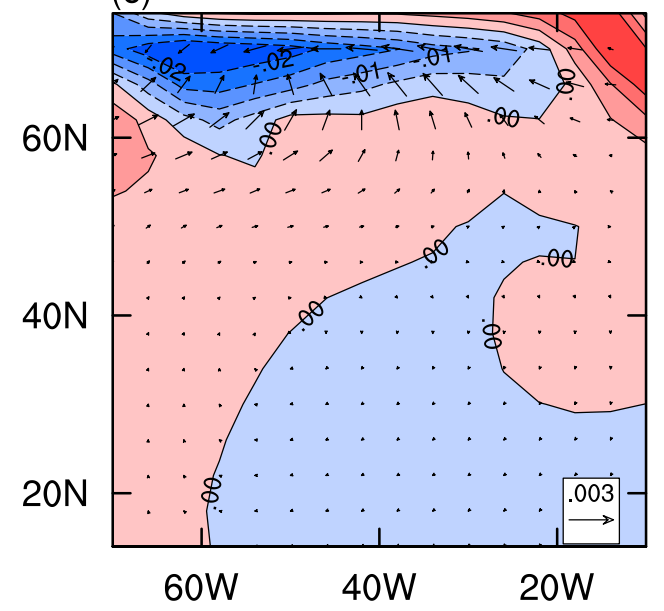

FIG. 6. (a) The convective zone in the non. conv. case (shaded area) and (b) the density difference between the cases of fully nonlinear and non. conv. on a longitude-depth section at $70^{\circ} \mathrm{N}$ at time $t=1.98$ years; (c) the horizontal (arrows) and vertical (lines) velocity difference between the cases of fully nonlinear and non. conv. in the surface layer in the year 1.98. The solid and dashed lines represent the positive and negative values, respectively, in (b) and (c).

velocity anomalies grow slower if $u^{\prime}\left(R_{0} \cos \phi\right)^{-1} \partial \rho^{\prime} / \partial \theta$ is removed, corresponding to the fact that the MOC decreases less with respect to the fully nonlinear case. Moreover, the time for the MOC to reach its minimum is shortened by about 3 years if removing $u^{\prime}\left(R_{0} \cos \phi\right)^{-1} \partial \rho^{\prime} / \partial \theta$.

As shown in Fig. 7, for a typical time slice $(t=7.17$ years), the upper-level-mean density anomalies show negative zonal gradients, and the corresponding velocity anomalies are negative north of $60^{\circ} \mathrm{N}$ in the $u^{\prime}\left(R_{0} \cos \phi\right)^{-1} \partial \rho^{\prime} / \partial \theta$ case (Figs. 7a,b). The process $u^{\prime}\left(R_{0} \cos \phi\right)^{-1} \partial \rho^{\prime} / \partial \theta$ in the fully nonlinear case represents the transport of water with lower density from the northeast to the northwest, which increases the zonal density gradient (Fig. 7c). Through the thermal wind relation, the increase of the zonal density gradient promotes a slowdown of the MOC. Moreover, $\rho_{W E}^{\prime}$ and MOC' reach a minimum when $\rho_{N S}^{\prime}$ and $\mathrm{MOC}^{\prime}$ reach zero during years $10-20$. The process $u^{\prime}\left(R_{0} \cos \phi\right)^{-1} \partial \rho^{\prime} / \partial \theta$ slows down the MOC further, and correspondingly, $\rho_{N S}^{\prime}$ is increased. The time for $\rho_{N S}^{\prime}\left(\rho_{N S}^{\prime}<0\right)$ and $\mathrm{ZOC}^{\prime}$ $\left(\mathrm{ZOC}^{\prime}<0\right)$ to reach zero is therefore shortened in the fully nonlinear case, with respect to the $u^{\prime}\left(R_{0} \cos \phi\right)^{-1} \partial \rho^{\prime} / \partial \theta$ (Fig. 7d). Hence, the time for $M O C^{\prime}$ and $\rho_{W E}^{\prime}$ to reach the minimum is also shortened.

During years 5-18, the $v^{\prime} R_{0}^{-1} \partial \rho^{\prime} / \partial \phi$ case shows significant difference from the fully nonlinear case, which is, however, only clearly seen in the objective function (Fig. 5b). After removing the nonlinear process $v^{\prime} R_{0}^{-1} \partial \rho^{\prime} / \partial \phi$, the upper-level velocities decrease, which 
(a)

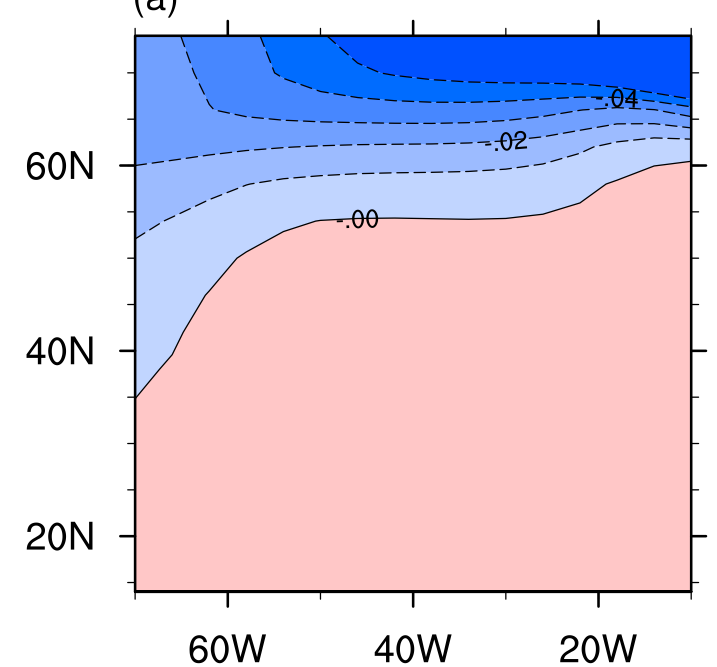

(c)

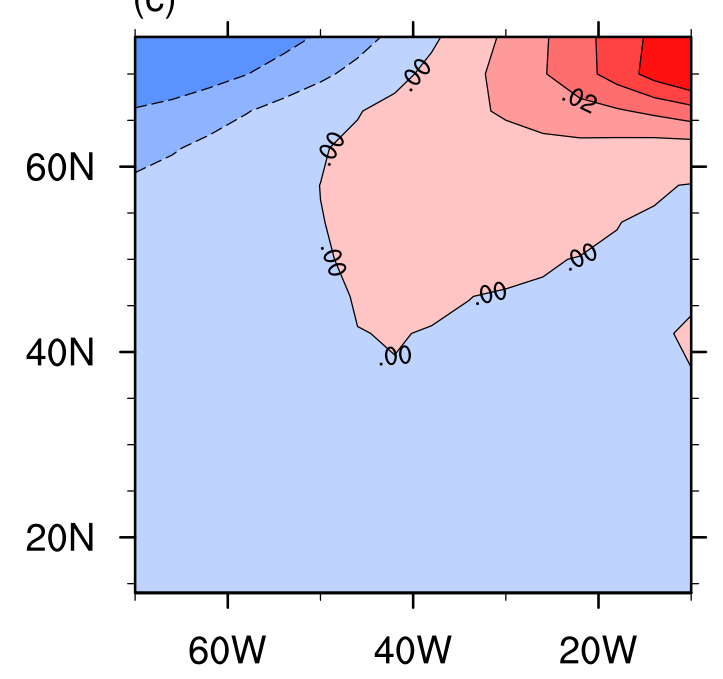

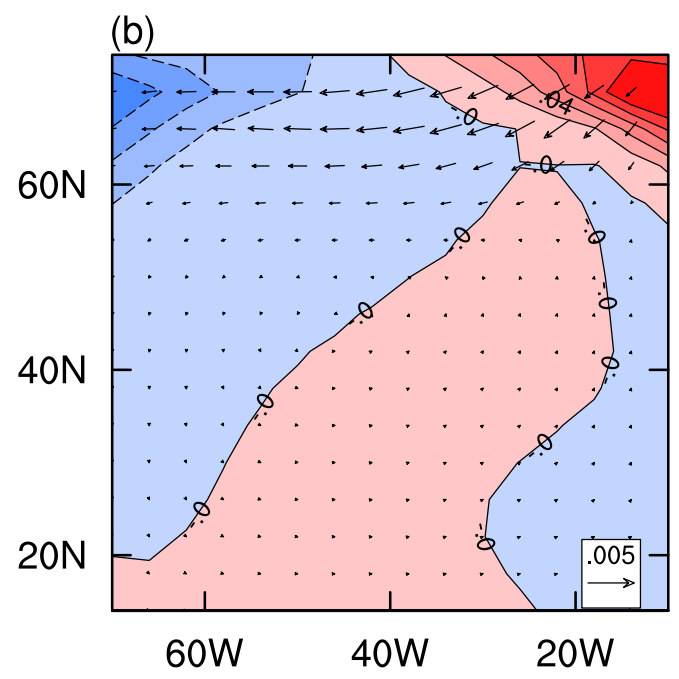

(d)

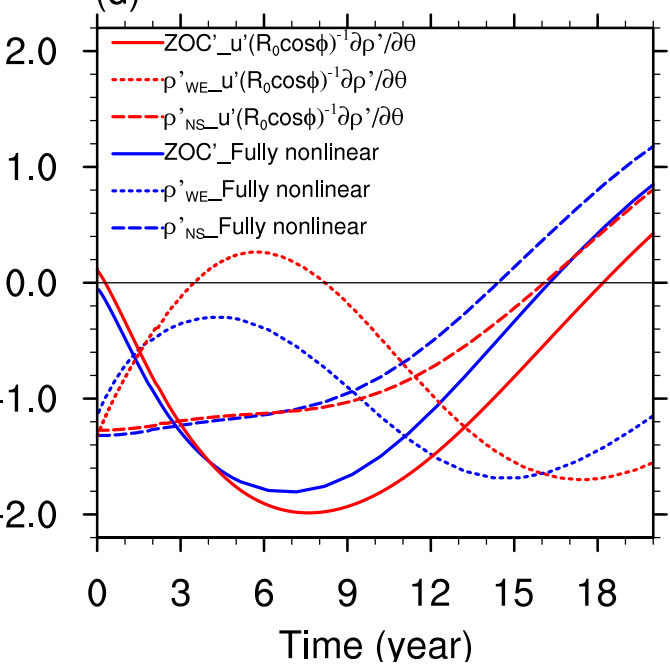

FIG. 7. The upper-level-mean (a) density anomalies and (b) velocity anomalies in the $u^{\prime}\left(R_{0} \cos \phi\right)^{-1} \partial \rho^{\prime} / \partial \theta$ case; (c) the upper-level-mean density difference between the cases of fully nonlinear and $u^{\prime}\left(R_{0} \cos \phi\right)^{-1} \partial \rho^{\prime} / \partial \theta$ in the year 7.17; and (d) the normalized indices of $\mathrm{ZOC}^{\prime}, \rho_{W E}^{\prime}$, and $\rho_{N S}^{\prime}$ in the $u^{\prime}\left(R_{0} \cos \phi\right)^{-1} \partial \rho^{\prime} / \partial \theta$ (red) and fully nonlinear cases (blue). The solid (dashed) lines denote the positive (negative) values in (a)-(c), and the arrows (lines) denote the horizontal (vertical) velocities in (b).

corresponds to the fact that the MOC decreases more with respect to the fully nonlinear case. Hence, the meridional advection of density anomalies represented by $v^{\prime} R_{0}^{-1} \partial \rho^{\prime} / \partial \phi$ strengthens the MOC. As shown in Fig. 8 for a typical time slice ( $t=7.17$ years), the upperlevel-mean density anomalies show a negative meridional gradient (Fig. 8a), while the meridional velocity presents a negative anomaly (Fig. 8b) in the $v^{\prime} R_{0}^{-1} \partial \rho^{\prime} / \partial \phi$ case. Therefore, the negative density anomalies are advected southward by this nonlinear process in the fully nonlinear case, leading to positive (negative) pressure anomalies south (north) of $70^{\circ} \mathrm{N}$ in the fully nonlinear case, with respect to the $v^{\prime} R_{0}^{-1} \partial \rho^{\prime} / \partial \phi$ case
(Fig. 8c). This negative anomaly of meridional pressure gradient strengthens the meridional velocities in the upper level (Fig. 8d). This process is a negative feedback, which inhibits the further slowdown of upper-level-mean meridional velocities. In addition, the contribution of $v^{\prime} R_{0}^{-1} \partial \rho^{\prime} / \partial \phi$ is dominant in the zonal belt of $70^{\circ} \mathrm{N}$, while the MMSF locates near $60^{\circ} \mathrm{N}$ (Fig. 1a). The MMSF changes because of the contribution of $v^{\prime} R_{0}^{-1} \partial \rho^{\prime} / \partial \phi$ are relatively small; therefore, the MMSF cannot represent this contribution.

Finally, the cases removing the nonlinear processes in the momentum equations all coincide with the fully nonlinear case (Figs. 5c,d). This suggests that the 
(a)

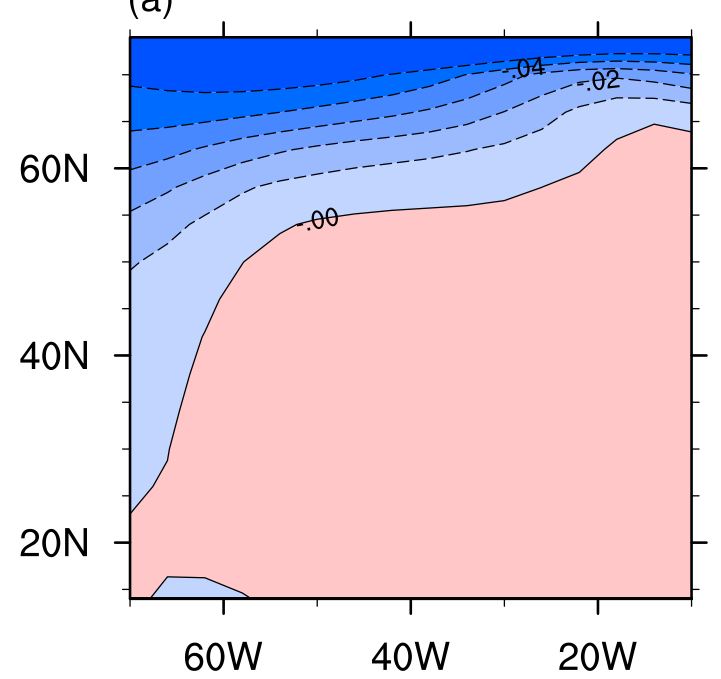

(c)

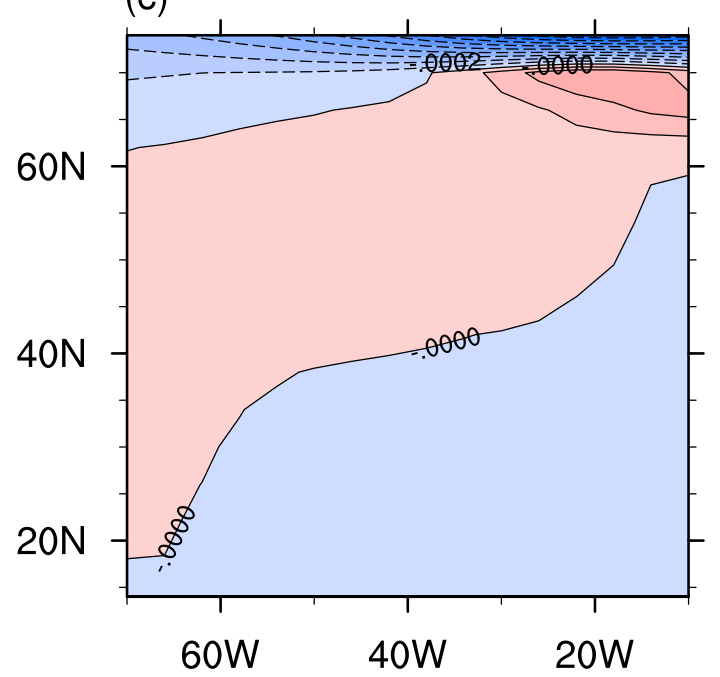

(b)

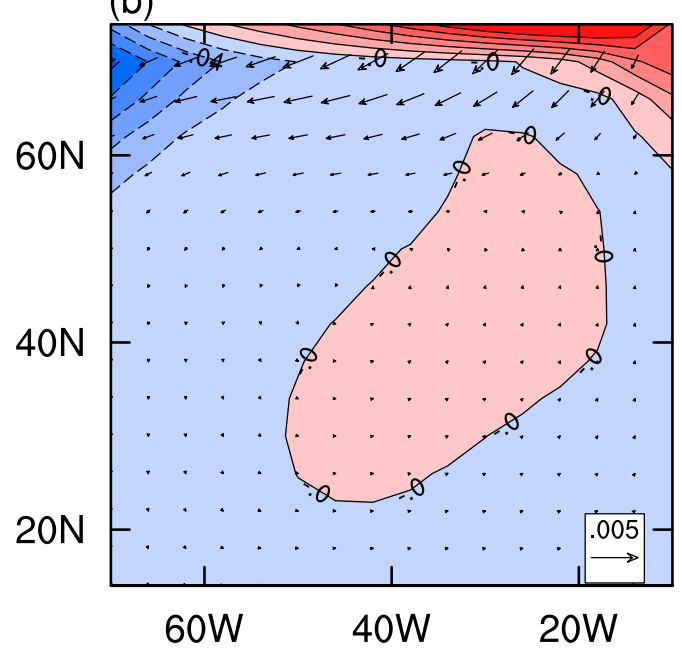

(d)

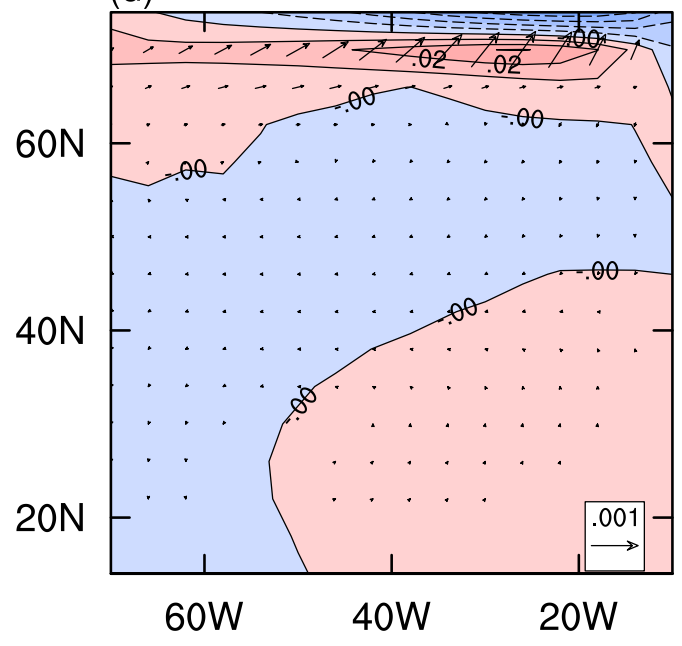

FIG. 8. The upper-level-mean (a) density anomalies and (b) velocity anomalies in the $v^{\prime} R_{0}^{-1} \partial \rho^{\prime} / \partial \phi$ case; the upperlevel-mean (c) pressure difference and (d) velocity difference between the cases of fully nonlinear and $v^{\prime} R_{0}^{-1} \partial \rho^{\prime} / \partial \phi$ in the year 7.17. The solid (dashed) lines denote the positive (negative) values in (a)-(d), and the arrows (lines) denote the horizontal (vertical) velocities in (b) and (d).

respective contribution of the nonlinear processes in the momentum equations is negligible.

\section{The comparison between the NOIP and LOIP}

For given optimization time, constraints, and objective function, the LOIPs are the fastest-growing perturbations of all the possible ones in tangent linear models, while the NOIPs grow fastest in fully nonlinear models. If the amplitude of perturbations is relatively small, such that the linear approximation is valid, the difference between the NOIP and LOIP should be also small. However, the difference will become more significant with increasing amplitude of the perturbations.
For the amplitude of perturbations used in section $2 \mathrm{~d}$, how much is the difference between the NOIP and LOIP, as well as the resulting AMV in the fully nonlinear model? To what extent do the LOIP and NOIP resemble each other? These questions will be discussed in this section.

Theoretically, the LOIP is independent on the perturbation amplitude, and hence it can be calculated under small constraints $\left(\delta_{\mathrm{SSS}}=1 \mathrm{psu}\right.$ and $\left.\delta_{\mathrm{SST}}=1^{\circ} \mathrm{C}\right)$ and then rescaled to the constraints radii used to compute the NOIPs $\left(\delta_{\mathrm{SSS}}=3.2 \mathrm{psu}\right.$ and $\left.\delta_{\mathrm{SST}}=18^{\circ} \mathrm{C}\right)$. In this case, the NOIP and LOIP have the same amplitude in the sense of (12) and (13). The optimization time was taken to be 10 years, which is the same as in the 
(a)

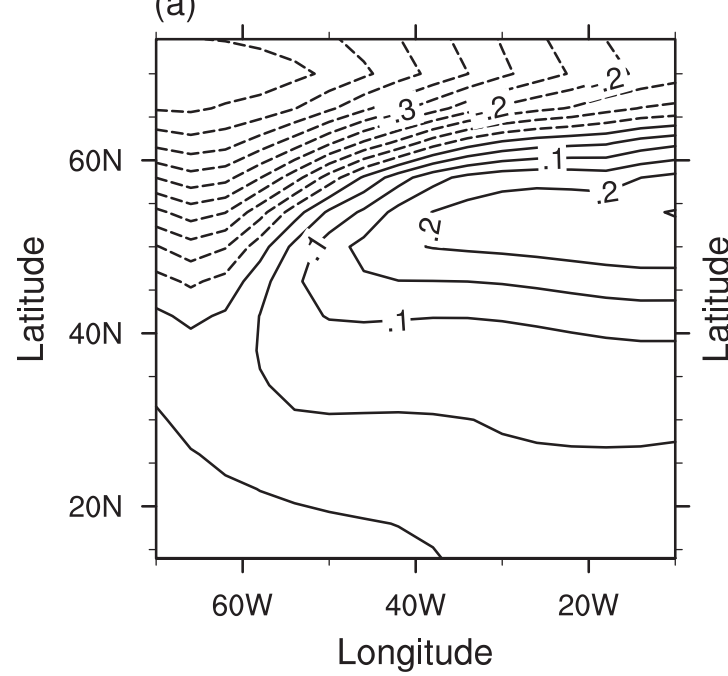

(c)

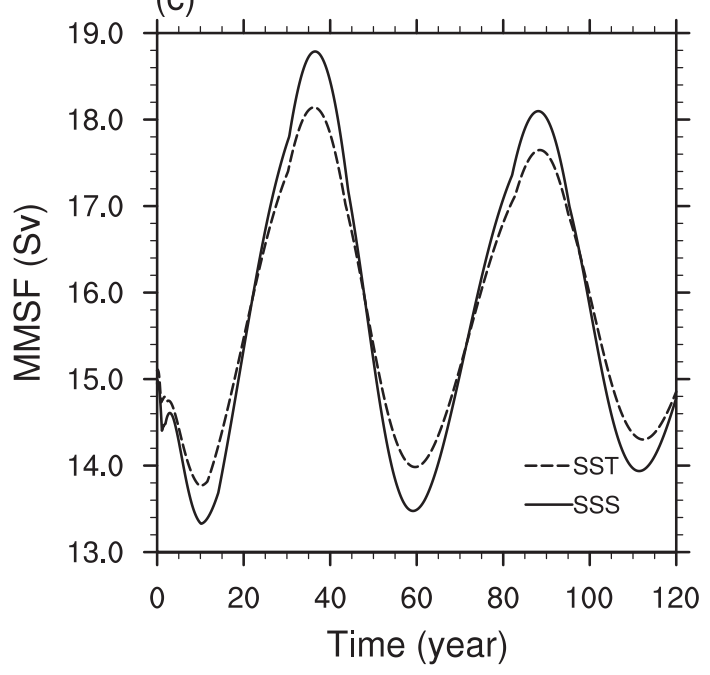

(b)

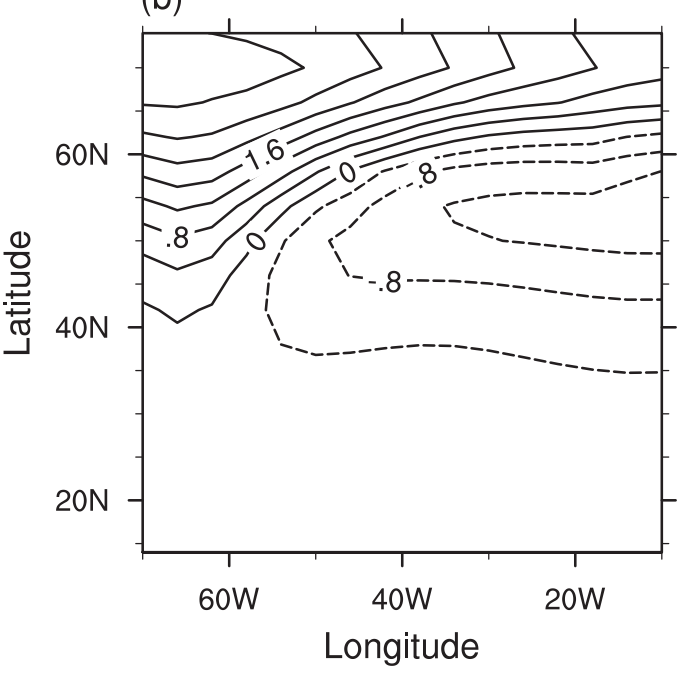

(d)

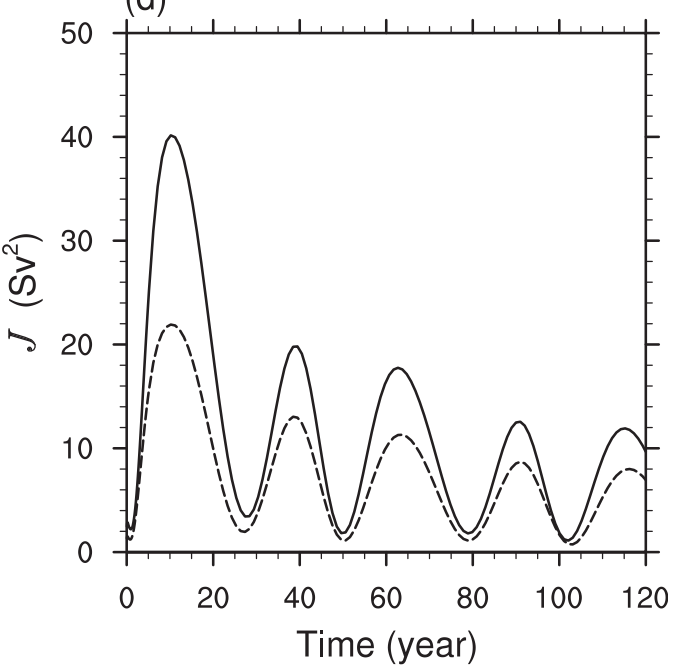

FIG. 9. As in Fig. 2, but for the FSV-type perturbations.

configurations for which the NOIPs were calculated (cf. Figs. 2a,b).

Both the SSS and SST anomalies of FSV type (Figs. 9a,b) show different patterns compared to those of the NOIPs (Figs. 2a,b). The SSS anomalies of the LOIP also have a strong meridional gradient, and the negative anomalies occur mainly in the northwestern corner (Fig. 9a). However, the center of negative anomalies moves westward, with respect to the NOIP anomalies (Fig. 2a). The positive anomalies appear over larger areas and the maximum $(0.25 \mathrm{psu})$ is larger than that of NOIP ones $(0.06 \mathrm{psu})$. The case of SST is similar: the anomalies of LOIP show relatively weaker positive anomalies and stronger negative ones (Fig. 9b), with respect to the NOIP ones (Fig. 2b).

Regarding adaptive observations, the observation system should be deployed in the areas where the perturbation amplitude is maximal. According to the LOIP results, the observation should be deployed in the northwestern part and the area of $\left[50^{\circ} \mathrm{N}, 60^{\circ} \mathrm{N}\right] \times\left[10^{\circ} \mathrm{W}, 40^{\circ} \mathrm{W}\right]$, whereas only the northwest is the priority according to the NOIP anomalies.

The multidecadal oscillations excited by the LOIP anomalies are similar to those by the NOIP ones but the amplitude of the oscillations is smaller (Figs. 9c,d). The MOC reaches a minimum around year 10 . For the case of NOIP SSS (SST) anomalies (Fig. 2c), this minimum is 12.25 Sv (13.31 Sv), while for the case of LOIP SSS (SST) anomalies (Fig. 9c), the minimum is $13.33 \mathrm{~Sv}(13.77 \mathrm{~Sv})$. The objective function also shows significant differences (Fig. 9d) between LOIP and NOIP perturbations. For the SSS anomalies, the maximum caused by the LOIP anomalies is about $1 / 3$ smaller than that by the NOIP 
ones. This indicates that LOIP perturbations can lead to an underestimation of MOC variations caused by the SST or SSS anomalies.

To further compare the differences between NOIP and LOIP perturbations, we calculated the NOIP and LOIP SSS anomalies under different amplitudes. Specifically, the NOIP were obtained under different constraint radii $\left(\delta_{\mathrm{Sss}}=1,2,3.2,4\right.$, and $\left.5 \mathrm{psu}\right)$ in (13), with an optimization time of $t=10$ years. As the pattern of the LOIP is independent on the constraint radius, we just calculated the LOIP under a small constraint radius $\left(\delta_{\mathrm{Sss}}=1 \mathrm{psu}\right)$ and then rescaled it to the corresponding constraint radii. To compare the difference between the patterns of the NOIP and LOIP perturbations, we calculated their normalized projection coefficient $P$ according to

$$
P\left(\mathbf{V}_{1}, \mathbf{V}_{2}\right)=\left(\mathbf{V}_{1} \cdot \mathbf{V}_{2}\right) / \sqrt{\left(\mathbf{V}_{1} \cdot \mathbf{V}_{1}\right)\left(\mathbf{V}_{2} \cdot \mathbf{V}_{2}\right)},
$$

where $\mathbf{V}_{1}$ and $\mathbf{V}_{2}$ represent the NOIP and LOIP, respectively.

As shown in Fig. 10a, the normalized projection coefficient decreases quickly as the constraint radius increases. For the perturbations with $\delta_{\mathrm{SsS}}=1$ and 2 psu that correspond to amplitudes (absolute maximum) of 0.18 and 0.34 psu, respectively (Fig. 10b), the LOIP and NOIP closely resemble each other. However, for $\delta_{\mathrm{sss}}=3.2,4$, and 5 psu that correspond to amplitudes of $0.49,0.57$, and 0.63 psu, respectively, the values of $P$ decrease with amplitude (i.e., 0.85, 0.76, and 0.64, respectively).

Figures $2 \mathrm{c}$ and $2 \mathrm{~d}$ present the MMSF and $J$ at the end of the optimization time, that is, 10 years, caused by the NOIP and LOIP. For $\delta_{\text {SSS }}=1$ and 2 psu, the MMSF difference remains relatively small, with errors of 0.03 and $0.26 \mathrm{~Sv}$, and the objective function $J$ also shows small relative differences of $0.9 \%$ and $4.6 \%$. It indicates that the linear approximation is valid for these initial perturbations. However, for the cases of $\delta_{\mathrm{sSs}}=3.2,4$, and $5 \mathrm{psu}$, the errors of MMSF increase substantially, that is $0.92,1.49$, and $2.18 \mathrm{~Sv}$, and the relative differences of $J$ are $17 \%, 29 \%$, and $48 \%$, respectively.

In summary, for the cases of $\delta_{\mathrm{Sss}}=1$ and $2 \mathrm{psu}$, the differences of NOIP and LOIP, as well as the resulting AMV are relatively small, and the linear approximation is valid. However, for the cases of $\delta_{\mathrm{sss}}=3.2,4$, and 5 psu, there exist significant differences between the patterns and the nonlinear evolutions of the NOIP and LOIP. Therefore, the linear approximation is problematic. For the case of $\delta_{\mathrm{sss}}=3.2 \mathrm{psu}$ that corresponds to an SSS anomaly of $0.5 \mathrm{psu}$ (an upper bound of the observed ones), the NOIP provides a better description than the LOIP of the nonlinear behavior of AMOC, the optimal thermohaline anomalies, and the resulting AMV.

\section{Summary and discussion}

Within a highly idealized three-dimensional ocean circulation model under prescribed heat and freshwater flux boundary conditions, the nonlinear optimal initial perturbations (NOIP) of SSS and SST exciting multidecadal AMOC variability were obtained using the CNOP method. In this model, the multidecadal variability is caused by internal ocean dynamics, which is one of the suggested mechanisms from GCM studies (Delworth and Mann 2000). The model is not able to represent any of the other mechanisms of multidecadal Atlantic variability that have been mentioned in the literature (Eden and Jung 2001; Timmermann et al. 1998; Jungclaus et al. 2005).

The physics of the excitation of AMOC variability by the NOIP was analyzed in detail. The NOIP with the typical amplitudes of observed SSS and SST variations correspond to strong (weak) meridional (zonal) density gradient anomalies with the absolute maximum in the northwest. This indicates that the northwestern basin contains most of the information of SSS and SST variations to improve the AMOC forecast skill over a lead time of 10 years. Therefore, the patterns of the NOIP can be considered as the optimal precursor, indicating where to make observations to improve the forecast skill of this variability (Duan et al. 2004). The optimal SSS and SST anomalies lead to reductions of the AMOC by 3.6 and $2.5 \mathrm{~Sv}$, respectively, after about 10 years. These provide the upper bounds of the AMOC variability caused by the instantaneous SSS and SST variations.

Under the prescribed heat and freshwater flux boundary conditions, the AMOC variability shows the characteristics of multidecadal oscillations with a period of about 50 years. After the first 30 years, the oscillations are dominated by linear physics, which involves the interaction of zonal and meridional density gradient anomalies and velocity anomalies through thermal wind relation, advection, and convection. However, during years $0-30$, nonlinear processes have an important influence on the AMOC strength. Convective processes strengthen the AMOC during years 0-2, the presence of zonal density advection promotes the slowdown of the AMOC during years 7-20 and shortens the time for the AMOC to reach its minimum, and meridional density advection inhibits the slowdown of meridional velocities in the upper ocean during years 5-18. In addition, the initial anomalies decay gradually to zero in time as the background flow is stable. That is why the contributions of the nonlinear processes are significant only in the first 30 years and insignificant after that.

The linear optimal initial perturbations (LOIP), for example, First Singular Vector (FSV), of SSS and SST 
(a)

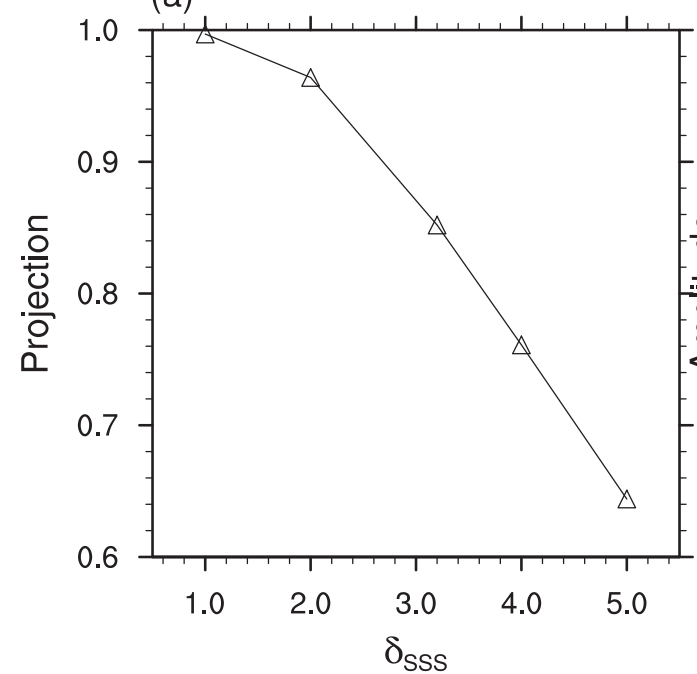

(c)

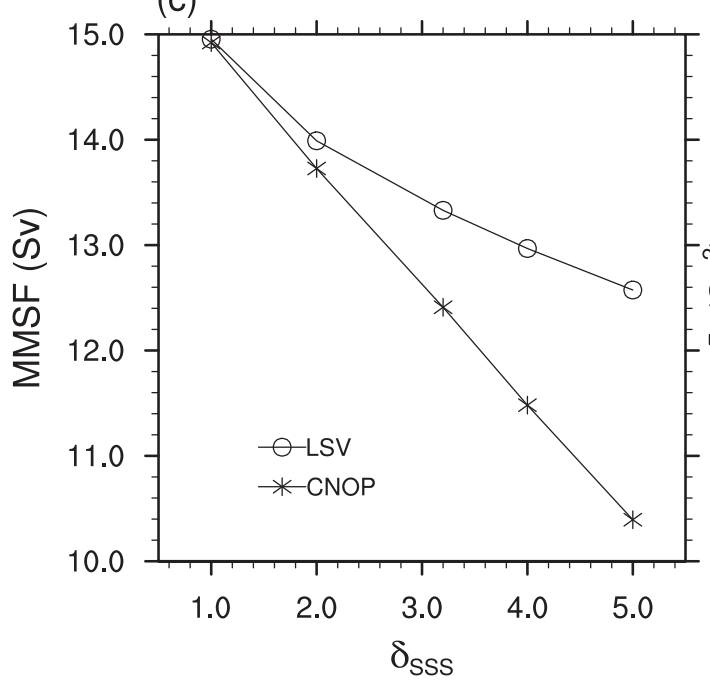

(b)

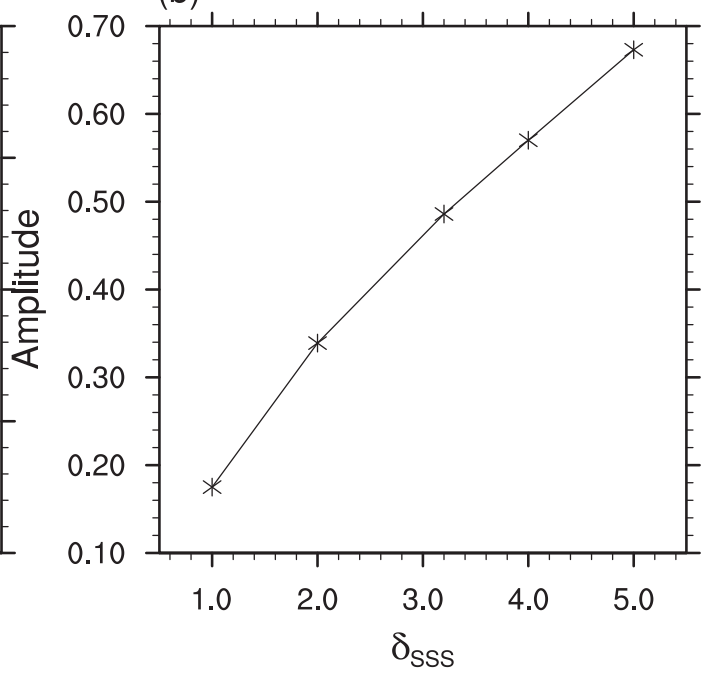

(d)

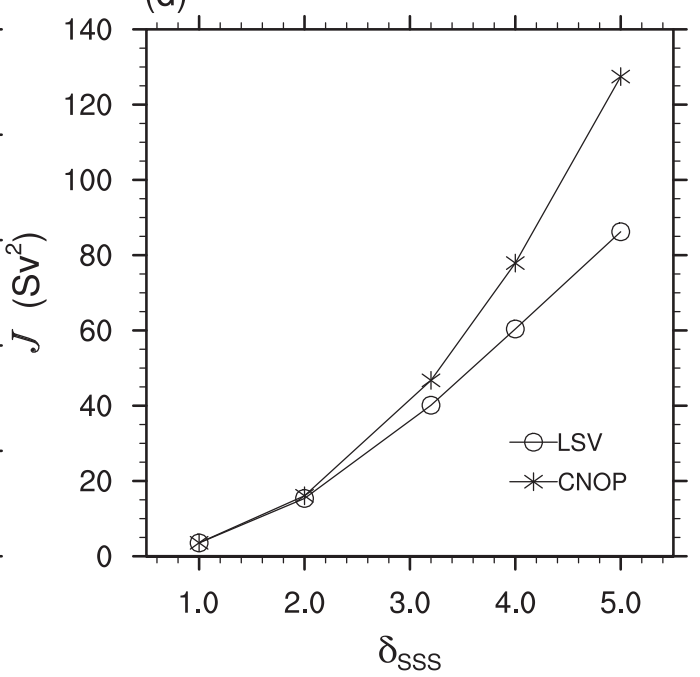

FIG. 10. (a) Projection coefficients of the optimal initial SSS perturbations of CNOP and FSV type vs the constraint radius; (b) the amplitude (absolute maximum) of CNOP-type perturbations vs the constraint radius; (c) the variations of MMSF; and (d) $J$ in the fully nonlinear model with a time delay of 10 years caused by optimal initial SSS perturbations of CNOP and FSV type with different constraint radii.

were also determined. These anomalies have different patterns than those of the NOIP. Taking the LOIP of SSS as an example, the center of the negative SSS anomalies moves westward and the positive anomalies occupy more areas with larger values, with respect to the NOIP. Based on the LOIP, from the view of adaptive observations, not only the northwestern basin but also the midlatitudes near the eastern boundary should be monitored. This error is caused by neglecting the effects of the nonlinear processes during the growth of the LOIP anomalies.

The LOIP also excites multidecadal oscillations with a period of about 50 years. However, the amplitude is smaller than that caused by the NOIP. For the case of NOIP SSS anomalies, the minimum of MMSF is $12.25 \mathrm{~Sv}$, that is, a reduction of $3.6 \mathrm{~Sv}$, while for the case of LOIP SSS anomalies, the minimum is only $13.33 \mathrm{~Sv}$, that is, a reduction of $2.5 \mathrm{~Sv}$. This indicates that the LOIP anomalies do not represent the correct response to perturbations in the nonlinear regime and that the CNOP method is more effective for the investigation of the excitation of AMOC multidecadal oscillations when using fully nonlinear models.

In this study, the patterns of the optimal thermohaline anomalies with relatively large amplitudes, as well as the bounds of the resulting AMOC variability, were 
determined. These anomalies can be considered as the optimal precursor (a kind of initial perturbations that can easiest lead to the concerned event), rather than the optimally growing initial error in predictability studies. In the linear regime, the patterns of the optimal precursor and the optimally growing initial error are theoretically identical, regardless of the basic state. However, in the nonlinear regime, they are not necessarily the same, since the NOIP is amplitude dependent (Wang et al. 2013).

For small amplitudes, LOIP and NOIP resemble each other and lead to similar variations of AMOC. As the amplitude increases, their similarity decreases quickly and the difference of resulting variations of AMOC increases substantially. For instance, for a typical amplitude of GSA events ( $0.5 \mathrm{psu})$, the value of normalized projection coefficient $P$ is 0.85 , the difference of MMSF is about $1 \mathrm{~Sv}$, and the relative difference of $J$ is $17 \%$. This suggests that for small amplitudes, such that the linear approximation is valid, the LOIP is still useful in the nonlinear framework to determine the optimally growing initial error and the optimal precursor. However, for relatively large amplitudes, there are significant differences between the LOIP and NOIP, and the NOIP provides a better description of the nonlinear behavior of the AMOC.

The AMOC multidecadal variability is here determined by internal factors, that is, the linear and nonlinear mechanisms, but it is also influenced by the external factors, that is, the instantaneous SST or SSS anomalies caused by the atmospheric forcing. Here it only provides a possible explanation on the excitation of AMOC multidecadal oscillation that is highly simplified. Through the idealized configurations, these results indicate that the observed oscillations may be attributed to some SSS or SST variations with short time scale and specific spatial pattern, rather than those with stochastic spatial patterns lasting for decades.

The optimal initial perturbations and the responses of the AMOC are dependent on the types of the boundary conditions. Under mixed boundary conditions the dominating mechanism is different. After superimposing the negative SSS anomalies at high latitudes, the AMOC slows down, and the temperature at high latitudes decreases, which compensates partly the decrease of the density caused by the SSS anomalies. However, the decrease of temperature is inhibited by the strong atmospheric damping in the mixed boundary conditions. Therefore, the response of the AMOC to the SSS anomalies is relatively strong under mixed boundary conditions. Correspondingly, NOIP SSS anomalies are also changed: the negative anomalies are relatively weak and strong positive anomalies appear in the western subbasin of $40^{\circ} \mathrm{N}$ ( $\mathrm{Zu}$ et al. 2013). Similar results have also been found by Sévellec et al. (2009) by calculating the LOIP.

The pattern of NOIP also depends on the optimization time. If the optimization time is set to be longer than 10 years, the maximum anomalies of the NOIP will move eastward. This indicates that for forecasts with different lead times, from the view of adaptive observations, the key observational areas will be different.

Although the NOIPs are determined here in an idealized ocean model, it is interesting to consider from observations where the largest variability in SST and SSS occurs in the North Atlantic. Actually, the SSS and SST in the northwestern basin, the Labrador Sea, display strong variability (Avsic et al. 2006; Robson et al. 2014). According to the results of this study, these thermohaline surface changes can lead to a strong modification of the AMOC in the future decade. In addition, the strength of the current AMOC variability may be attributed largely to the thermohaline surface changes that occurred 10 years ago in the northwestern basin (Robson et al. 2014). Of course, there are many other processes, not represented in our model, which may influence the NOIP and the AMOC response. These are variability in the wind forcing, the nonlinear processes in the equation of state, and the geometry and the bathymetry of the basin. However, these effects are outside the scope of the present paper and are left for future work.

Acknowledgments. The authors thank the anonymous reviewers for their useful suggestions. This study was supported by the National Natural Scientific Foundation of China (41230420), the Strategic Priority Research Program of the Chinese Academy of Sciences (XDA11010303), the NSFC Shandong Joint Fund for Marine Science Research Centers (U1406401), and China Special Fund for Meteorological Research in the Public Interest (GYHY201306018). The work of H.D. was supported by the Netherlands Organization for Scientific Research (NWO) through the COMPLEXITY project PreKurs and the Netherlands Earth System Science Center.

\section{REFERENCES}

Alexander, J., and A. H. Monahan, 2009: Nonnormal perturbation growth of pure thermohaline circulation using a $2 \mathrm{D}$ zonally averaged model. J. Phys. Oceanogr., 39, 369-386, doi:10.1175/ 2008JPO3847.1.

Avsic, T., J. Karstensen, U. Send, and J. Fischer, 2006: Interannual variability of newly formed Labrador Sea Water from 1994 to 2005. Geophys. Res. Lett., 33, L21S02, doi:10.1029/2006GL026913.

Belkin, I. M., S. Levitus, J. Antonov, and S. A. Malmberg, 1998: "Great Salinity Anomalies" in the North Atlantic. Prog. Oceanogr., 41, 1-68, doi:10.1016/S0079-6611(98)00015-9.

Birgin, E. G., J. M. Martínez, and M. Raydan, 2000: Nonmonotone spectral projected gradient methods on convex sets. SIAM J. Optim., 10, 1196-1211, doi:10.1137/S1052623497330963. 
Chen, F., and M. Ghil, 1995: Interdecadal variability of the thermohaline circulation and high-latitude surface fluxes. J. Phys. Oceanogr., 25, 2547-2568, doi:10.1175/1520-0485(1995)025<2547: IVOTTC $>2.0 . \mathrm{CO} ; 2$.

Colin de Verdière, A., and T. Huck, 1999: Baroclinic instability: An oceanic wavemaker for interdecadal variability. J. Phys. Oceanogr., 29, 893-910, doi:10.1175/1520-0485(1999)029<0893: BIAOWF $>2.0 . \mathrm{CO} ; 2$.

Cunningham, S., and Coauthors, 2007: Temporal variability of the Atlantic Meridional Overturning circulation at $26.5^{\circ} \mathrm{N}$. Science, 317, 935-938, doi:10.1126/science.1141304.

Delworth, T. L., and M. E. Mann, 2000: Observed and simulated multidecadal variability in the Northern Hemisphere. Climate Dyn., 16, 661-676, doi:10.1007/s003820000075.

_ - S. Manabe, and R. J. Stouffer, 1995: North Atlantic interdecadal variability in a coupled model. Natural Climate Variability on Decade-to-Century Time Scales, National Academies Press, 432-439.

de Niet, A., F. Wubs, A. T. van Scheltinga, and H. A. Dijkstra, 2007: A tailored solver for bifurcation analysis of oceanclimate models. J. Comput. Phys., 227, 654-679, doi:10.1016/ j.jcp.2007.08.006.

Dijkstra, H. A., 2007: Characterization of the multiple equilibria regime in a global ocean model. Tellus, 59A, 695-705, doi:10.1111/ j.1600-0870.2007.00267.x.

— , 2013: Nonlinear Climate Dynamics. Cambridge University Press, 367 pp.

—, H. Oksuzoglu, F. W. Wubs, and E. F. F. Botta, 2001: A fully implicit model of the three-dimensional thermohaline ocean circulation. J. Comput. Phys., 173, 685-715, doi:10.1006/ jcph.2001.6908.

_ L. T. Raa, M. Schmeits, and J. Gerrits, 2006: On the physics of the Atlantic multidecadal oscillation. Ocean Dyn., 56, 36-50, doi:10.1007/s10236-005-0043-0.

Duan, W., and M. Mu, 2006: Investigating decadal variability of El Nino-Southern Oscillation asymmetry by conditional nonlinear optimal perturbation. J. Geophys. Res., 111, C07015, doi:10.1029/2005JC003458.

— Applications to stability, sensitivity, and predictability. Sci. China, 52D, 883-906, doi:10.1007/s11430-009-0090-3.

,$- \ldots$, and B. Wang, 2004: Conditional nonlinear optimal perturbations as the optimal precursors for El Nino-Southern Oscillation events. J. Geophys. Res., 109, D23105, doi:10.1029/ 2004JD004756.

Eden, C., and T. Jung, 2001: North Atlantic interdecadal variability: Oceanic response to the North Atlantic Oscillation (1865-1997). J. Climate, 14, 676-691, doi:10.1175/1520-0442(2001)014<0676: NAIVOR $>2.0 . \mathrm{CO} ; 2$.

Enfield, D. B., A. M. Mestas-Nuñez, and P. J. Trimble, 2001: The Atlantic multidecadal oscillation and its relation to rainfall and river flows in the continental US. Geophys. Res. Lett., 28 , 2077-2080, doi:10.1029/2000GL012745.

Farrell, B. F., and P. J. Ioannou, 1996: Generalized stability theory-I: Autonomous operators. J. Atmos. Sci., 53, 2025-2040, doi:10.1175/ 1520-0469(1996)053<2025:GSTPIA > 2.0.CO;2.

Hawkins, E. D., and R. Sutton, 2009: Decadal predictability of the Atlantic Ocean in a coupled GCM: Forecast skill and optimal perturbations using linear inverse modeling. J. Climate, 22, 3960-3978, doi:10.1175/2009JCLI2720.1.

Hobbs, W. R., and J. K. Willis, 2012: Midlatitude North Atlantic heat transport: A time series based on satellite and drifter data. J. Geophys. Res., 117, C01008, doi:10.1029/2011JC007039.
Huck, T., and G. K. Vallis, 2001: Linear stability analysis of threedimensional thermally-driven ocean circulation: Application to interdecadal oscillations. Tellus, 53A, 526-545, doi:10.1111/ j.1600-0870.2001.00526.x.

Huisman, S. E., M. den Toom, H. A. Dijkstra, and S. Drijfhout, 2010: An indicator of the multiple equilibria regime of the Atlantic Meridional Overturning Circulation. J. Phys. Oceanogr., 40, 551-567, doi:10.1175/2009JPO4215.1.

Jungclaus, J. H., H. Haak, M. Latif, and U. Mikolajewicz, 2005: Arctic-North Atlantic interactions and multidecadal variability of the meridional overturning circulation. J. Climate, 18, 4013-4031, doi:10.1175/JCLI3462.1.

Kennedy, J. J., N. A. Rayner, R. O. Smith, M. Saunby, and D. E. Parker, 2011: Reassessing biases and other uncertainties in sea-surface temperature observations since 1850: 1. Measurement and sampling errors. J. Geophys. Res., 116, D14103, doi:10.1029/ 2010JD015218.

Kerswell, R. R., C. C. T. Pringle, and A. P. Willis, 2014: An optimization approach for analysing nonlinear stability with transition to turbulence in fluids as an exemplar. Rep. Prog. Phys., 77, 085901, doi:10.1088/0034-4885/77/8/085901.

Kushnir, Y., 1994: Interdecadal variations in North Atlantic sea surface temperature and associated atmospheric conditions. J. Climate, 7, 141-157, doi:10.1175/1520-0442(1994)007<0141: IVINAS $>2.0 . \mathrm{CO} ; 2$.

Manabe, S., and R. J. Stouffer, 1997: Coupled ocean-atmosphere model response to freshwater input: Comparison to Younger Dryas Event. Paleoceanography, 12, 321-336, doi:10.1029/96PA03932.

Monahan, A. H., J. Alexander, and A. J. Weaver, 2008: Stochastic models of the meridional overturning circulation: time scales and patterns of variability. Philos. Trans. Roy. Soc., 366A, 2525-2542, doi:10.1098/rsta.2008.0045.

Mu, M., W. Duan, and B. Wang, 2003: Conditional nonlinear optimal perturbation and its applications. Nonlinear Processes Geophys., 10, 493-501, doi:10.5194/npg-10-493-2003.

_ - L. Sun, and H. A. Dijkstra, 2004: The sensitivity and stability of the ocean's thermohaline circulation to finite-amplitude perturbations. J. Phys. Oceanogr., 34, 2305-2315, doi:10.1175/ 1520-0485(2004)034<2305:TSASOT>2.0.CO;2.

—, W. Duan, H. Xu, and B. Wang, 2006: Applications of conditional nonlinear optimal perturbation in predictability study and sensitivity analysis of weather and climate. Adv. Atmos. Sci., 23, 992-1002, doi:10.1007/s00376-006-0992-3.

- H. Xu, and W. Duan, 2007: A kind of initial errors related to "spring predictability barrier" for El Nino events in ZebiakCane model. Geophys. Res. Lett., 34, L03709, doi:10.1029/ 2006 GL027412.

_ - F. Zhou, and H. Wang, 2009: A method for identifying the sensitive areas in targeted observations for tropical cyclone prediction: Conditional nonlinear optimal perturbation. Mon. Wea. Rev., 137, 1623-1639, doi:10.1175/2008MWR2640.1.

Qin, X. H., and M. Mu, 2012: Influence of conditional nonlinear optimal perturbations sensitivity on typhoon track forecasts. Quart. J. Roy. Meteor. Soc., 138, 185-197, doi:10.1002/qj.902.

Robson, J., D. Hodson, E. Hawkins, and R. Sutton, 2014: Atlantic overturning in decline? Nat. Geosci., 7, 2-3, doi:10.1038/ngeo2050.

Sévellec, F., and A. V. Fedorov, 2015: Optimal excitation of AMOC decadal variability: links to the subpolar ocean. Prog. Oceanogr., 132, 287-304, doi:10.1016/j.pocean.2014.02.006. , T. Huck, M. Ben Jelloul, and J. Vialard, 2009: Nonnormal multidecadal response of the thermohaline circulation induced by optimal surface salinity perturbations. J. Phys. Oceanogr., 39, 852-872, doi:10.1175/2008JPO3998.1. 
Sun, G., and M. Mu, 2011: Response of a grassland ecosystem to climate change in a theoretical model. Adv. Atmos. Sci., 28, 1266-1278, doi:10.1007/s00376-011-0169-6.

Sun, L., M. Mu, D. J. Sun, and X. Y. Yin, 2005: Passive mechanism of decadal variation of thermohaline circulation. J. Geophys. Res., 110, C07025, doi:10.1029/2005JC002897.

te Raa, L. A., and H. A. Dijkstra, 2002: Instability of the thermohaline ocean circulation on interdecadal timescales. J. Phys. Oceanogr., 32, 138-160, doi:10.1175/1520-0485(2002)032<0138: IOTTOC $>2.0 . \mathrm{CO} ; 2$.

Timmermann, A., and G. Lohmann, 2000: Noise-induced transitions in a simplified model of the thermohaline circulation. J. Phys. Oceanogr., 30, 1891-1900, doi:10.1175/1520-0485(2000)030<1891: NITIAS $>2.0 . \mathrm{CO} ; 2$.

_- M. Latif, and R. Voss, 1998: Northern Hemispheric interdecadal variability: A coupled air-sea mode. J. Climate, 11, 1906-1931, doi:10.1175/1520-0442-11.8.1906.

Tziperman, E., and P. J. Ioannou, 2002: Transient growth and optimal excitation of thermohaline variability. J. Phys. Oceanogr., 32, 3427-3435, doi:10.1175/1520-0485(2002)032<3427: TGAOEO $>2.0 . \mathrm{CO} ; 2$.

Wang, C., S. Dong, and E. Munoz, 2010: Seawater density variations in the North Atlantic and the Atlantic meridional overturning circulation. Climate Dyn., 34, 953-968, doi:10.1007/ s00382-009-0560-5.

Wang, Q., M. Mu, and H. A. Dijkstra, 2012: Application of the conditional nonlinear optimal perturbation method to the predictability study of the Kuroshio large meander. Adv. Atmos. Sci., 29, 118-134, doi:10.1007/s00376-011-0199-0.

,$- \ldots$, and -2013 : The similarity between optimal precursor and optimally growing initial error in prediction of Kuroshio large meander and its application to targeted observation. J. Geophys. Res. Oceans, 118, 869-884, doi:10.1002/ jgrc.20084.

Wang, Y., S. Li, and D. Luo, 2009: Seasonal response of Asian monsoonal climate to the Atlantic Multidecadal Oscillation. J. Geophys. Res., 114, D02112, doi:10.1029/ 2008JD010929.

Zanna, L., P. Heimbach, A. M. Moore, and E. Tziperman, 2012: Upper-ocean singular vectors of the North Atlantic climate with implications for linear predictability and variability. Quart. J. Roy. Meteor. Soc., 138, 500-513, doi:10.1002/qj.937.

Zu, Z., M. Mu, and H. A. Dijkstra, 2013: Optimal nonlinear excitation of decadal variability of the North Atlantic thermohaline circulation. Chin. J. Oceanol. Limnol., 31, 1368-1374, doi:10.1007/s00343-014-3051-4. 